\title{
Lehrkräfteeinschätzungen zu Unterrichtsqualität mit digitalen Medien: Zusammenhänge zur wahrgenommenen technischen Schulausstattung, Medienunterstützung, digitalen Kompetenzselbsteinschätzungen und Wertüberzeugungen
}

\author{
Jennifer Quast • Charlott Rubach $(\mathbb{D} \cdot$ Rebecca Lazarides \\ Eingegangen: 31. Oktober 2020 / Überarbeitet: 18. Juni 2021 / Angenommen: 18. Juni 2021 / Online \\ publiziert: 14. Juli 2021 \\ (C) Der/die Autor(en) 2021
}

Zusammenfassung Obwohl der Einsatz digitaler Medien in Lehr-Lern-Prozessen zunehmend an Bedeutung gewinnt, befassen sich Studien nur vereinzelt mit der Frage, inwiefern digitale Medien im Unterricht zur Umsetzung von Unterrichtsqualitätsdimensionen genutzt werden. Gleichzeitig ist wenig über die Bedingungsfaktoren einer solchen qualitätsvollen Umsetzung von Unterricht mit digitalen Medien bekannt. Die vorliegende Studie untersucht vor diesem Hintergrund, inwiefern Schulmerkmale vermittelt über Lehrkräftemerkmale dazu beitragen, dass Lehrkräfte digitale Medien nutzen, um im Unterricht zu strukturieren, kognitiv zu aktivieren, konstruktiv zu unterstützen sowie zu individualisieren. Ausgewertet wurden Daten von 280 Lehrkräften an Schulen der Sekundarstufe in Deutschland (52,1\% weiblich, Alter: $M=43,88, \mathrm{SD}=10,00)$. Die Ergebnisse des Strukturgleichungsmodells verweisen darauf, dass die Zufriedenheit mit der schulischen Unterstützung zum Einsatz digitaler Medien vermittelt über die digitalen (berufsunabhängigen) Kompetenzselbsteinschätzungen in positivem Zusammenhang mit der Nutzung digitaler Medien zum Zwecke der Individualisierung, kognitiven Aktivierung und konstruktiven Unterstützung im Unterricht steht. Die von Lehrkräften berichtete vielfältige technische Schulausstattung sowie die berichteten positiven Wertüberzeugungen in Bezug auf den Einsatz digitaler Medien im Unterricht sind direkt positiv mit der

Die beiden Autorinnen J. Quast und C. Rubach teilen sich die Erstautorenschaft.

\section{Jennifer Quast}

Zentrum für Qualitätsentwicklung in Lehre und Studium, Universität Potsdam, Potsdam, Deutschland

Dr. Charlott Rubach

School of Education, University of California, Irvine, Irvine, CA, USA

E-Mail: crubach@uci.edu

Prof. Dr. Rebecca Lazarides $(\triangle)$

Arbeitsbereich Schulpädagogik m.d.S. Schul- und Unterrichtsentwicklung, Department

Erziehungswissenschaft, Universität Potsdam, Karl-Liebknecht-Str. 24-25, 14476 Potsdam,

Deutschland

E-Mail: rebecca-lazarides@uni-potsdam.de 
Nutzung digitaler Medien zur Umsetzung der Unterrichtsqualitätsdimensionen assoziiert.

Schlüsselwörter Unterrichtsqualität · Digitale Medien · Lehrkräfte ·

Wertüberzeugungen · Digitale Kompetenzselbsteinschätzungen

\title{
Teaching quality with ICT from teachers' perspectives: Associations with school ICT equipment, perceived ICT support, and teachers' ICT competence and value beliefs
}

\begin{abstract}
Information and communications technology (ICT) are of high importance for school-related learning and teaching processes. However, studies rarely have examined whether teachers use ICT to implement principles of teaching quality in their classrooms. Furthermore, little is known under which conditions ICT contributes to high-quality teaching. Against this background, this study investigates to what extent school characteristics indirectly relate to teachers' ICT use for structuring classrooms, cognitive activation, constructive support and individualization in class through teacher characteristics. The study uses the data of $N=280$ German secondary school teachers $(52.1 \%$ female, age: $M=43.88, S D=10.00)$. Results of structural equation model indicate that teachers' satisfaction with ICT school support is positively related to the ICT use for individualization, cognitive activation and constructive support in class through teachers' (basic) ICT competence beliefs. ICT school equipment reported by teachers and teachers' positive ICT value beliefs are directly positively associated with the ICT use for teaching quality in class.
\end{abstract}

Keywords Teaching quality ICT $\cdot$ Teachers $\cdot$ Value beliefs $\cdot$ ICT competence beliefs

\section{Einleitung}

Befunde der International Computer and Information Literacy Study (ICILS) zeigen, dass sich zwischen ICILS 2013 und ICILS 2018 der Anteil der befragten Lehrkräfte, die täglich digitale Medien im Unterricht einsetzen, mehr als verdoppelte (Drossel et al. 2019) und dass Schulen den Einsatz digitaler Medien zunehmend als wichtig ansehen (Gerick et al. 2019). Der Einsatz digitaler Medien im Unterricht zielt darauf $a b$, die Unterrichtsqualität zu erhöhen und fachspezifische Lernergebnisse der Lernenden zu fördern sowie den kompetenten Umgang der Lernenden mit digitalen Medien zu verbessern (Eickelmann und Gerick 2020). Um diese Ziele zu erreichen, sollten Lehrkräfte digitale Medien auf pädagogisch wirksame Weise in Unterrichtskonzepte einbinden (Heldt et al. 2020). Bislang befassen sich Forschungsarbeiten nur vereinzelt mit der Frage, inwiefern digitale Medien im Unterricht dafür eingesetzt werden, Unterrichtsprozesse qualitätsvoll zu gestalten (Backfisch et al. 2021; Lucas et al. 2021). Die vorliegende Studie adressiert diese Forschungslücke und untersucht, inwieweit Lehrkräfte digitale Medien zur Umsetzung von Unterrichtsprozessqualität nutzen (Klieme 2019). 
Gleichzeitig befasst sich die vorliegende Studie mit den Bedingungsfaktoren der Nutzung digitaler Medien. Empirische Befunde verweisen auf die Wichtigkeit von Schulmerkmalen sowie individuellen Merkmalen der Lehrkräfte (Drossel et al. 2019; Sundqvist et al. 2021). Sundqvist et al. (2021) verdeutlichen zudem, dass Schulmerkmale (z.B. die Unterstützung durch die Schule zum Einsatz digitaler Medien) vermittelt über Lehrkräftemerkmale (z. B. empfundene Nützlichkeiten zum Einsatz digitaler Medien) mit dem Einsatz digitaler Medien in Zusammenhang stehen. Ein zweites Ziel der vorliegenden Studie ist die Validierung dieser Befunde mit Daten aus Deutschland. Der Mehrwert unserer Studie für die Forschung zu digitalen Medien in Unterrichtsprozessen ist folglich, dass internationale Befunde repliziert werden können. Damit wird ein Beitrag dazu geleistet, valide Erkenntnisse zu Bedingungsfaktoren der Nutzung digitaler Medien zur Umsetzung qualitätsvoller Unterrichtsprozesse $\mathrm{zu}$ identifizieren. Unsere Befunde tragen zum aktuellen Kenntnisstand bei, indem sie aufzeigen, wie Lehrkräfte digitale Medien zur Umsetzung von Unterrichtsqualitätsdimensionen nutzen und welche spezifischen Schul- und Lehrkräftemerkmale für die Umsetzung von Bedeutung sind. Weiterhin liegt der Mehrwert für die Schulund Unterrichtsforschung darin, Prozesse des Zusammenwirkens von Schulmerkmalen und der Nutzung digitaler Medien zur Umsetzung qualitätsvollen Unterrichts aufzuzeigen.

\section{Theoretischer Hintergrund}

\subsection{Unterrichtsqualität und digitale Medien}

Ein etabliertes Modell qualitätsvollen Unterrichtens ist das der drei generischen Basisdimensionen von Unterrichtsqualität (Klieme 2019), welches drei Dimensionen von Unterrichtsqualität unterscheidet: Klassenführung, kognitive Aktivierung und konstruktive Unterstützung. Diese Basisdimensionen gelten als fachunspezifisch. Studien zeigen, dass die Dimension Klassenführung keine fachspezifischen Anteile aufweist, während das unterstützende Unterrichtsklima allerdings fachspezifische Anteile aufweist (Praetorius et al. 2016). Die Basisdimensionen der Unterrichtsqualität werden als Tiefenstrukturen des Unterrichts konzeptualisiert - also als Strukturen des Unterrichts, die im Sinne einer Prozessqualität von Unterricht Lernen, Verstehen, Motivation und Ausdauer begünstigen (Klieme 2019). Methoden und Lernarrangements des Unterrichts (z. B. Arbeitsformen, Medien, Gesprächsformate) werden demgegenüber als Sichtstrukturen des Unterrichts bezeichnet. Klieme (2020) beschreibt dabei den Einsatz digitaler Medien als ,Sonderfall des Methodenspektrums“.

Verschiedene aktuelle Arbeiten befassen sich mit den theoretischen Verknüpfungen von Unterrichtsqualitätsdimensionen und digitalen Medien (Klieme 2020; Voss und Wittwer 2020) und untersuchen empirisch die Nutzung digitaler Medien für die Umsetzung der Unterrichtsqualität (Backfisch et al. 2021). Angenommen wird, dass mit der Umsetzung digitaler Lernformate die Veränderung und Neugestaltung von Lehr-Lernprozessen einhergeht und digitale Medien didaktische Potenziale für die Prozessqualität des Unterrichts haben (Eickelmann und Gerick 2020). Unter 
qualitätsvollem Unterrichten mit digitalen Medien wird in der vorliegenden Studie die Nutzung digitaler Medien zur Umsetzung der Prozessqualität des Unterrichts verstanden. Es wird davon ausgegangen, dass entsprechend unterrichtstheoretischer Angebot-Nutzungs-Modelle digitale Medien die Umsetzung eines qualitätsvollen Unterrichtsangebots der Unterrichtsprozessqualität ermöglichen (z. B. strukturierte Unterrichtsgestaltung durch automatisierte Lernabläufe, Förderung des kollaborativen Lernens durch digitale Medien). Gleichzeitig nehmen wir an, dass die Effektivität des Einsatzes digitaler Medien im Unterricht wesentlich von den kognitiven, motivationalen und verhaltensbezogenen Nutzungsprozessen seitens der Lernenden abhängt. Ausgehend von diesem Verständnis wird in der vorliegenden Studie untersucht, inwiefern Lehrkräfte digitale Medien zur Umsetzung von Unterrichtsqualitätsdimensionen im Sinne eines qualitätsvollen Unterrichtsangebots nutzen.

Die Dimension der Klassenführung bezieht sich auf Strategien der Lehrkraft zur Steuerung des Unterrichts, zum Umgang mit Unterrichtsstörungen und zur Strukturierung der Unterrichtsinhalte, die zur effektiven Nutzung der Unterrichtszeit beitragen (Klieme 2006). Digitale Medien unterstützen eine effiziente Klassenführung beispielsweise durch die Möglichkeit der vereinfachten Strukturierung von Lerninhalten (Sieve 2015) sowie durch Möglichkeiten systematischer Zusammenfassungen durch digitale Visualisierungen (Sabanci et al. 2014). Zudem können digitale Medien eingesetzt werden, um verschiedene mediale Formate zusammenzufügen und damit eine übersichtliche Organisation von Unterrichtsinhalten und -materialien zu ermöglichen (Akçayır und Akçayır 2017).

Kognitive Aktivierung umfasst anspruchsvolle und anwendungsorientierte Aufgaben, die an Vorwissen der Lernenden anknüpfen (Praetorius et al. 2018) sowie Möglichkeiten der selbstständigen Entwicklung und Begründung von Lösungswegen (Baumert et al. 2004). Digitale Medien tragen durch verstärkte Anwendungsorientierung behandelter Aufgaben zur kognitiven Aktivierung bei (Kerres 2018). Befunde des Länderindikators 2017 verweisen zudem darauf, dass digitale Medien im Unterricht zur selbstständigen Recherche, Aufbereitung und Visualisierung von Unterrichtsinhalten eingesetzt werden können (Eickelmann et al. 2017). Zur vertiefenden Auseinandersetzung mit Unterrichtsinhalten werden weiterhin Simulationsprogramme genutzt, welche die Kommunikation über Unterrichtsinhalte zwischen Schülerinnen und Schülern und Algorithmen ermöglichen (Xu et al. 2021). Zudem werden digitale Medien zur Erarbeitung, Veranschaulichung und Reflexion von Unterrichtsinhalten (z. B. in digitalen Laboren, Kornilov und Khanina 2020) sowie zur Reflexion eigener Lernvorgänge (z. B. Videoaufnahmen eigener Vorträge; Erstellung von Erklärvideos) eingesetzt.

Konstruktive Unterstützung beschreibt die Begleitung von Lernprozessen und die Unterstützung der Lernenden bei Verständnisschwierigkeiten durch die Lehrkraft (Dubberke et al. 2008). Digitale Medien können in diesem Sinne zur Bereitstellung personalisierten Feedbacks (Levy 2009; Ware et al. 2012) sowie zum Monitoring des individuellen Lernfortschritts eingesetzt werden (Faber 2020; Hillmayr et al. 2017).

Individualisierung wird neben den drei Basisdimensionen von Unterrichtsqualität als weiteres Merkmal erfolgreichen Unterrichts beschrieben (Lipowsky 2015). Diskutiert wird, inwieweit digitale Medien hilfreich bei der Umsetzung der Individua- 
lisierung im Unterricht sind und den Umgang mit Heterogenitätsstrukturen fördern (siehe Heinen und Kerres 2015). Digitale Medien bieten insbesondere für die individuelle Lernunterstützung vielfältige Umsetzungsmöglichkeiten (Eickelmann 2010). Mithilfe digitaler Medien und algorithmischer Strukturen lassen sich Lerninhalte und -aufgaben adaptiv an die individuellen Voraussetzungen der Lernenden anpassen, beispielsweise durch multimediale Materialien (Heinen und Kerres 2015), interaktive Aufgabenstellungen, die Bereitstellung individualisierter Lösungswege und interaktiven, individuellen Feedbacks (Schaumburg 2017).

\subsection{Bedingungsfaktoren des Einsatzes digitaler Medien}

Zur Erklärung des individuellen Handelns und individueller Entscheidungsprozesse kann das Erwartungs-Wert-Modell von Eccles et al. (1983) herangezogen werden. Für das Verständnis der Nutzung digitaler Medien zur Gestaltung qualitätsvoller Unterrichtsprozesse von Lehrkräften hat das Modell Bedeutung, da es aufzeigt, dass individuelle motivationale Überzeugungen, demografische Merkmale sowie Einstellungen der Lehrkräfte und zur Verfügung stehende Ressourcen der beruflichen Umwelt das Unterrichtshandeln von Lehrkräften bedingen können. Ein weiteres zentrales Modell zur Beschreibung der Voraussetzungen kompetenten digitalen Handelns im Unterricht ist das will, skill, tool model of technology integration (Knezek und Christensen 2016). Das Modell postuliert, dass persönliche Wertüberzeugungen, Kompetenzen und Kompetenzselbsteinschätzungen sowie Schulausstattungsmerkmale den Einsatz digitaler Medien in Lehr-Lern-Prozessen prägen.

\subsubsection{Wertüberzeugungen zum Einsatz digitaler Medien}

Persönliche Überzeugungen zum Einsatz digitaler Medien sind ein zentraler Einflussfaktor für das Unterrichtshandeln (Knezek und Christensen 2016). Lehrkräfte mit einer positiven Einstellung gegenüber digitalen Medien berichten im Vergleich zu Lehrkräften mit weniger positiven Einstellungen häufiger davon, digitale Medien im Unterricht einzusetzen (Ertmer et al. 2012; Sundqvist et al. 2021). Lehrkräfte, die nur wenige Bedenken gegenüber neuen Technologien äußern, berichten auch, digitale Medien häufiger und vielfältiger im Unterricht einzusetzen (Aslan und Zhu 2016; Rahimi und Yadollahi 2011).

Das Erwartungs-Wert-Modell (Eccles et al. 1983) differenziert Wertüberzeugungen in persönliche Wichtigkeit, Interesse, Nützlichkeit und Kosten. Persönliche Wichtigkeit beschreibt die identitätsbezogene Bedeutung der Aktivität, beispielsweise inwiefern Lehrkräfte den Einsatz digitaler Medien als persönlich bedeutsam für ihre Berufsidentität empfinden. Interesse bezieht sich auf die erwartete Freude in Zusammenhang mit einer Handlung. Internationale Befunde zeigen, dass Lehrkräfte, die Interesse am Einsatz digitaler Medien im Unterricht berichten, auch angeben, digitale Medien häufiger einzusetzen (Ertmer et al. 2012; Hew und Brush 2007). Nützlichkeit bezieht sich auf den empfundenen Nutzen einer Handlung für das Erreichen persönlicher Ziele. Empirische Befunde verweisen auf einen positiven Zusammenhang zwischen der von (angehenden) Lehrkräften empfundenen Nützlichkeit digitaler Medien im Unterricht und der von Lehrkräften berichteten 
Häufigkeit des Einsatzes digitaler Medien im Unterricht (Backfisch et al. 2021; Sundqvist et al. 2021). Kosten beziehen sich auf aufgabenbezogene psychologische Kosten und zeitlichen Aufwand (Eccles 2005). Ergebnisse von Sipilä (2011) zeigen, dass Lehrkräfte, die häufig digitale Medien im Unterricht nutzen, auch weniger psychologische Kosten empfinden.

\subsubsection{Digitale Kompetenzselbsteinschätzungen}

Digitale Kompetenzüberzeugungen von Lehrkräften können unter anderem in grundlegende (berufsunabhängige) und pädagogische (berufsbezogene) digitale Kompetenzüberzeugungen differenziert werden (Krumsvik 2014; Rubach und Lazarides 2021a). Diese Überzeugungen stehen in Zusammenhang mit der Implementierung von digitalen Medien in schulbezogene Lernumgebungen (O. Hatlevik 2017; Klassen und Chiu 2010). Häufig wird analysiert, inwiefern Lehrkräfte mit höheren pädagogischen (berufsbezogenen) digitalen Kompetenzüberzeugungen davon berichten, digitale Medien in Lehr-Lern-Prozesse zu implementieren (Guggemos und Seufert 2021; I. Hatlevik und Hatlevik 2018; Sailer et al. 2021). Weniger häufig wird sich mit der Frage beschäftigt, inwieweit Lehrkräfte auch über Kompetenzüberzeugungen verfügen müssen, welche den grundlegenden (berufsunabhängigen) Kompetenzbereichen wie Kommunikation und Kollaboration, Schützen und sicher Agieren oder Problemlösen und Handeln zuzuordnen sind (Rubach und Lazarides 2021a; O. Hatlevik 2017; Li et al. 2019). Angenommen wird jedoch, dass sowohl hohe Kompetenzüberzeugungen in grundlegenden (berufsunabhängigen) Bereichen als auch in pädagogischen (berufsbezogenen) Bereichen dazu beitragen, dass Lehrkräfte digitale Medien qualitätsvoll in Lehr-Lern-Prozessen implementieren (O. Hatlevik 2017; I. Hatlevik und Hatlevik 2018; Mishra und Koehler 2006).

\subsubsection{Technische Schulausstattung und schulische Unterstützung bei der Nutzung digitaler Medien}

Empirische Studien zeigen, dass eine bessere technische Schulausstattung in Zusammenhang mit der Nutzungshäufigkeit digitaler Medien im Unterricht steht (Drossel et al. 2017; Lucas et al. 2021). Weiterhin zeigt sich, dass die Unterstützung durch die Schule beim Einsatz digitaler Medien und die themenspezifische Kooperation im Kollegium positiv mit dem von Lehrkräften berichteten Einsatz digitaler Medien im Unterricht zusammenwirken (Lucas et al. 2021; Sundqvist et al. 2021). Die Unterstützung durch die Schule beim Einsatz digitaler Medien beinhaltet hierbei die technische und medienpädagogische Unterstützung durch die Schule sowie die persönliche Unterstützung durch Schulleitung und Kollegium. Auch die von der Schule gesetzten Bildungsziele und medienpädagogischen Konzepte wurden als Bedingungsfaktoren des Einsatzes von digitalen Medien identifiziert (Drossel et al. 2019; Lucas et al. 2021). Im Vergleich dieser Merkmale zeigt sich, dass die technische Ausstattung, Medienkonzepte und der Austausch im Kollegium gleichwertig zu dem von Lehrkräften berichteten Einsatz digitaler Medien beitragen (Drossel et al. 2019). Gleichzeitig verdeutlichen Studien, dass im Vergleich zur technischen Schulausstattung die konkrete Unterstützung durch die Schule beim Einsatz digitaler 
Medien eine größere Bedeutung für den Einsatz digitaler Medien innehat (Sundqvist et al. 2021; Amhag et al. 2019).

Nur vereinzelt untersuchen Studien das Zusammenwirken zwischen Schulmerkmalen und Lehrkräftemerkmalen in schulischen Digitalisierungsprozessen (Inan und Lowther 2010; Sundqvist et al. 2021). Befunde von Sundqvist et al. (2021) legen nahe, dass Schulmerkmale vermittelt über Lehrkräftemerkmale mit dem Einsatz digitaler Medien in Unterrichtsprozessen zusammenwirken. In der Studie steht die Zufriedenheit mit der schulischen Unterstützung zum Einsatz digitaler Medien in positivem Zusammenhang mit der von Lehrkräften berichteten Nützlichkeit zum Einsatz von Medien, welche dann wiederum mit dem Einsatz digitaler Medien in Lehr-Lern-Settings assoziiert ist (Sundqvist et al. 2021). Dabei wurden als abhängige Variablen die Nutzung digitaler Medien für die unterrichtsbezogenen kooperativen Lernprozesse, die Unterstützung der Lernprozesse von Lernenden sowie die Nutzung digitaler Medien zur Organisation und Unterrichtsplanung untersucht. Damit werden Annahmen zum Erwartungs-Wert-Modell von Eccles et al. (1983) bestätigt, dass Umweltfaktoren über motivationale Überzeugungen der Akteure individuelle Verhaltensweisen bedingen.

\subsubsection{Soziodemografische Merkmale der Lehrkräfte und Strukturmerkmale der Schule}

Soziodemografische Merkmale von Lehrkräften, wie Geschlecht und Berufserfahrung, sowie Strukturmerkmale der Schule, beispielsweise die Schulart, sind ebenfalls wichtige Wirkfaktoren für den unterrichtsbezogenen Einsatz digitaler Medien (GilFlores et al. 2017; Schmid et al. 2017). Befunde legen nahe, dass Lehrer im Vergleich zu Lehrerinnen häufiger digitale Medien im Unterricht einsetzen (Drossel et al. 2017; Tezci 2010). Lucas et al. (2021) zeigen auf, dass Lehrer im Vergleich zu Lehrerinnen häufiger digitale Medien für die methodische und pädagogische Unterrichtsgestaltung sowie Prüfungsgestaltung und -abnahme einsetzen. Gleichzeitig konnten keine geschlechtsspezifischen Unterschiede in der Umsetzung der konstruktiven Unterstützung und Individualisierung mithilfe digitaler Medien identifiziert werden (Lucas et al. 2021). Internationale Studien nahe legen, dass sich Lehrer im Vergleich zu Lehrerinnen kompetenter im Umgang mit digitalen Medien einschätzen (Rohatgi et al. 2016; Scherer et al. 2017). Differenziert betrachtet bestätigt sich auch für Lehrkräfte aus Deutschland, dass Lehrer sich im Vergleich zu Kolleginnen kompetenter in den Kompetenzbereichen Produzieren und Präsentieren, Schützen und sicher Agieren, Problemlösen und Handeln sowie Analysieren einschätzen (Rubach und Lazarides 2021a).

Auch die Berufserfahrung ist von Bedeutung für die Nutzung digitaler Medien im Unterricht - Lehrkräfte mit höherer Berufserfahrung integrieren digitale Medien seltener als Lehrkräfte mit geringer Berufserfahrung in ihren Unterricht (Gil-Flores et al. 2017; Tezci 2010). Angenommen wird, dass ältere Lehrkräfte mit höherer Berufserfahrung unsicherer im Umgang mit neuen digitalen Technologien sind und diese daher seltener einsetzen (López-Vargas et al. 2017).

Untersuchungen in Deutschland verdeutlichen zudem, dass Gymnasien gegenüber anderen Schulformen der Sekundarstufe technisch besser ausgestattet sind und 
dass Gymnasiallehrkräfte digitale Medien vielseitiger im Unterricht nutzen (Schmid et al. 2017; Wetterich, Burghart und Rave 2014). Befunde der ICILS-Studie 2018 zeigen (Schaumburg et al. 2019), dass das Nutzungsverhalten der Schülerinnen und Schüler sich zwischen Schulformen unterscheidet: Lernende am Gymnasium berichten weniger häufig, digitale Medien in der Schule für schulbezogene Zwecke zu nutzen, aber gleichzeitig auch weniger häufig, niemals digitale Medien für solche Zwecke zu benutzen. Hier stellt sich die Frage, inwieweit sich Angaben der Lernenden und der Lehrkräfte decken und Lehrkräfte am Gymnasium weniger häufig digitale Medien im Unterricht zur Umsetzung der Unterrichtsqualitätsdimensionen nutzen.

\subsection{Die vorliegende Studie}

Digitalisierung kann als Wandlungsprozess verstanden werden - digitale Technologien ergänzen und modernisieren analoge Unterrichtsprozesse und können Lehrund Lernprozesse verändern (Irion und Scheiter 2018; Kerres 2018). Allerdings ist wenig darüber bekannt, inwiefern Lehrkräfte digitale Medien nutzen, um Unterricht im Sinne einer hohen Prozessqualität aktivierend, strukturiert, individualisierend und unterstützend zu gestalten. Die vorliegende Studie befasst sich mit der Frage, inwiefern - aus Sicht von Lehrkräften - digitale Medien zur Strukturierung, kognitiven Aktivierung, konstruktiven Unterstützung und zur Individualisierung eingesetzt werden. Ein weiteres Forschungsinteresse ist, Faktoren zu identifizieren, die mit der Umsetzung qualitätsvollen Unterrichtens mit digitalen Medien in Zusammenhang stehen. In der vorliegenden Studie wird dieses Desiderat adressiert und untersucht, inwieweit Schulmerkmale vermittelt über Lehrkräftemerkmale mit der Nutzung digitaler Medien zur Umsetzung von Unterrichtsqualitätsdimensionen assoziiert sind. Das angenommene Modell ist in Abb. 1 dargestellt. Wir untersuchen die folgenden Forschungsfragen und Hypothesen:

FF1 Können die theoretisch und empirisch definierten Unterrichtsqualitätsdimensionen empirisch in einer Vier-Faktorenstruktur abgebildet werden?

H1 Angenommen wird, dass sich folgende vier latente Faktoren abbilden lassen: kognitive Aktivierung, Strukturierung, Individualisierung und konstruktive Unterstützung (Klieme 2019; Lipowsky 2006).

FF2 Inwieweit nutzen Lehrkräfte digitale Medien, um die Prozessqualität des Unterrichts in Bezug auf vier Unterrichtsqualitätsdimensionen umzusetzen?

H2 Explorativ wird angenommen, dass digitale Medien häufiger dafür eingesetzt werden, den Unterricht zu strukturieren und im Unterricht zu unterstützen sowie Lehr-Lern-Prozesse zu individualisieren. Wir gehen davon aus, dass digitale Medien seltener dazu eingesetzt werden, Prozesse der kognitiven Aktivierung zu implementieren. Ausgangspunkt der Annahme ist, dass Prozesse der kognitiven Aktivierung digital schwieriger umsetzbar sein könnten (Klieme 2020). 


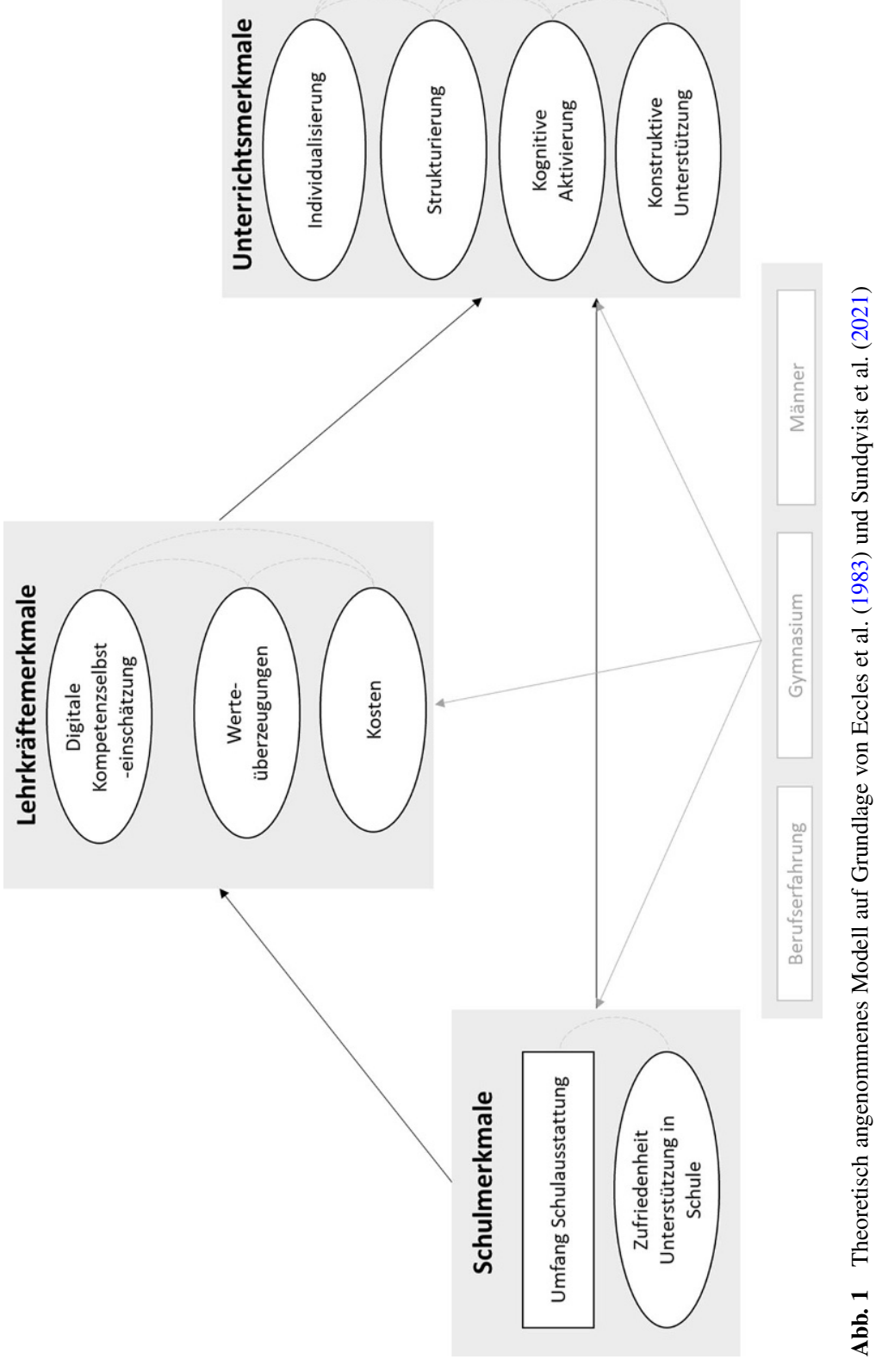


FF3 Inwieweit sind Schulmerkmale (technische Schulausstattung, Zufriedenheit mit der schulischen Unterstützung zur Nutzung digitaler Medien) indirekt über Lehrkräftemerkmale (digitale Kompetenzselbsteinschätzungen, Wertüberzeugungen, Kosten) mit der Nutzung digitaler Medien zur Umsetzung der Unterrichtsqualitätsdimensionen (kognitive Aktivierung, konstruktive Unterstützung, Individualisierung, Strukturierung) assoziiert?

H3 Digitale Kompetenzselbsteinschätzungen sowie Wertüberzeugungen zum Einsatz digitaler Medien sind positiv mit der selbstberichteten Nutzung digitaler Medien zur Umsetzung der Unterrichtsqualitätsdimensionen assoziiert (siehe Amhag et al. 2019; Sundqvist et al. 2021).

H4 In Anlehnung an Befunde von Sipilä (2011) wird angenommen, dass die Kostenüberzeugungen zum Einsatz digitaler Medien negativ mit der Nutzung digitaler Medien zur Umsetzung von Unterrichtsqualitätsdimensionen assoziiert sind.

H5 Auf Grundlage theoretischer Annahmen (Eccles et al. 1983) und empirischer Befunde (Sundqvist et al. 2021) wird angenommen, dass die Zufriedenheit mit der schulischen Unterstützung zur Nutzung digitaler Medien sowie die technische Schulausstattung indirekt über Lehrkräftemerkmale (digitalen Kompetenzselbsteinschätzungen, Werte- und Kostenüberzeugungen zum Einsatz digitaler Medien) mit der selbstberichteten Nutzung digitaler Medien zur Umsetzung der Unterrichtsqualitätsdimensionen assoziiert sind.

FF4 Inwiefern erklären sozio-demografische Merkmale von Lehrkräften und Strukturmerkmale der Schule sowohl untersuchte Schulmerkmale (technische Schulausstattung, Zufriedenheit mit der schulischen Medienunterstützung), Lehrkräftemerkmale (digitale Kompetenzselbsteinschätzungen, Werte- und Kostenüberzeugungen) sowie die Nutzung digitaler Medien zur Umsetzung von Unterrichtsqualitätsdimensionen (kognitive Aktivierung, konstruktive Unterstützung, Individualisierung, Strukturierung)?

H6 Lehrer nutzen im Vergleich zu Lehrerinnen häufiger digitale Medien zur Umsetzung der Unterrichtsqualitätsdimensionen und schätzen sich als digital kompetenter ein (Lucas et al. 2021).

H7 Lehrkräfte mit geringerer Berufserfahrung nutzen im Vergleich zu Lehrkräften mit höherer Berufserfahrung häufiger digitale Medien zur Umsetzung der Unterrichtsqualitätsdimensionen und schätzen sich als digital kompetenter ein (Gil-Flores et al. 2017; López-Vargas et al. 2017).

H8 Gymnasiallehrkräfte nutzen im Vergleich zu Lehrkräften anderer Sekundarschularten weniger digitale Medien zur Umsetzung der Unterrichtsqualitätsdimensionen (siehe Schaumburg et al. 2019). 


\section{Methode}

\subsection{Stichprobe}

Für die vorliegende Studie wurden Daten von $n=280$ Lehrkräften (52,1\% weiblich) aus der DigiKompEL-Studie (Rubach und Lazarides 2017-2019; https://www. researchgate.net/project/DigiKompEL-Einschaetzung-digitaler-Kompetenzen-beiLehramtsstudierenden-und-Lehrkraeften) genutzt, in deren Kontext Lehrkräfte via Twitter und eines Newsletters des Fortbildungsunternehmens Fobizz im Befragungszeitraum von Januar bis Februar 2019 an einer 30-minütigen Online-Befragung teilnahmen. In der Studie wurde eine Teilstichprobe $(N=449)$ jener Lehrkräfte genutzt, deren Fragebogendaten zu mindestens $75 \%$ vollständig vorlagen $(n=337)$ und die an einer Schule der Sekundarstufe tätig waren $(n=280)$. Die Analyse fehlender Werte durch stichprobengrößenadjustierte $t$-Tests für unabhängige Stichproben (Bonferroni-Korrektur) zeigte, dass sich die Lehrkräfte, für die mehr als $75 \%$ der Daten vorlagen, in den für diese Studie genutzten Variablen nicht signifikant von den Lehrkräften unterschieden, deren Angaben zu weniger als $75 \%$ vorlagen.

Die Lehrkräfte in der Teilstichprobe unterrichteten an Schulen in allen deutschen Bundesländern ${ }^{1}$. Das durchschnittliche Alter dieser Lehrkräfte lag bei 43,88 Jahren $(S D=10,00)$, die durchschnittliche Berufserfahrung bei 13,78 Jahren $(S D=9,87)$. Etwas weniger als die Hälfte der Lehrkräfte in der Teilstichprobe $(42,5 \%)$ arbeiteten an einem Gymnasium und etwas mehr als die Hälfte (57,5\%) an einer anderen Schulform der Sekundarstufe I und II. Etwa zwei Drittel der Lehrkräfte in der Teilstichprobe $(69,3 \%)$ waren Vollzeit beschäftigt. Die am häufigsten unterrichteten Fächer waren Informatik (35,7\%), Mathematik (28,9\%), Deutsch (21,4\%), Englisch $(20,4 \%)$, Geschichte $(14,3 \%)$, Chemie $(12,1 \%)$, Biologie $(10,7 \%)$ und Physik $(10,7 \%) .^{2}$

Beachtet werden muss, dass die Stichprobe spezifische Charakteristika aufweist, die bei der Interpretation der Befunde berücksichtigt werden müssen. Durch die Art der Erhebung ist es denkbar, dass eine spezifische Gruppe von Lehrkräften angesprochen wurde, nämlich Lehrkräfte, die sich aktiv mit den Prozessen der Digitalisierung im schulischen Kontext auseinandersetzen. Die Spezifität der Stichprobe wird auch daran deutlich, dass mehr als ein Drittel der Befragten Informatik als Schulfach unterrichten. Zur zusätzlichen Einordnung der genutzten Stichprobe wurde ein Vergleich zur Gesamtpopulation der Lehrkräfte in Deutschland (Statistisches Bundesamt 2019) vorgenommen. Es zeigt sich, dass in der Stichprobe männliche Lehrkräfte überproportional vertreten sind.

\footnotetext{
1 18,6\% aus Nordrhein-Westfalen, 12,9\% Niedersachsen, 12,5\% aus Baden-Württemberg, 12,5\% aus Berlin, 8,9\% aus Bayern, 5,7\% aus Schleswig-Holstein, 5,4\% aus Brandenburg, 5,0\% aus Hamburg, 5,0\% aus Hessen, 3,6\% aus Sachsen, 2,5\% aus Thüringen, 1,4\% aus Mecklenburg-Vorpommern, 1,4\% aus Saarland, 1,1\% aus Rheinland-Pfalz, 1,1\% aus Sachsen-Anhalt, 0,7\% aus Bremen, und 1,8\% fehlende Angaben.

$222,1 \%$ der befragten Lehrkräfte gaben an, ein anderes, nicht im Fragebogen erfasstes Fach zu unterrichten.
} 


\subsection{Messinstrumente}

Die Items aller Skalen sind in Tab. 5 im Anhang gelistet.

\subsubsection{Digitale Medien und Unterrichtsqualitätsdimensionen}

Die Nutzung digitaler Medien zur Umsetzung der Unterrichtsqualitätsdimensionen wurde in Anlehnung an Klieme (2020) und Lipowsky (2006) durch vier Skalen erfasst: Strukturierung, Kognitive Aktivierung, Konstruktive Unterstützung und Individualisierung. Daher wurden Originalskalen für die vorliegende Studie dahingehend umformuliert, dass gemessen wurde, ob und wie oft Strukturierung und konstruktive Unterstützung (Rakoczy und Klieme 2005), kognitive Aktivierung (Kunter et al. 2008) und Individualisierung (Clausen 2002) mithilfe digitaler Medien umsetzt werden. Eine fünfstufige Antwortskala wurde genutzt $(1=$ in keiner Stunde, $2=$ in sehr wenigen Stunden, 3=in einigen Stunden, 4=in den meisten Stunden, 5=in jeder Stunde). Informationen zur Reliabilität und Validität der Skalen sind im Ergebnisteil der Studie beschrieben (Abschn. 4.1).

\subsubsection{Zufriedenheit mit der schulischen Unterstützung zum Einsatz digitaler Medien}

Anhand mehrerer Items bewerteten Lehrkräfte ihre Zufriedenheit mit den Rahmenbedingungen zum Einsatz digitaler Medien an ihrer Schule. Dazu gehören beispielsweise der Umfang und die Qualität der IT-Ausstattung sowie verschiedene Formen der Unterstützung bei der Nutzung digitaler Medien. In Anlehnung an Sundqvist et al. (2021) wird in dieser Studie die Bedeutung der Zufriedenheit mit der schulischen Unterstützung zur Nutzung digitaler Medien untersucht. Daher wurden drei Items ausgewählt, die erfassen, wie zufrieden Lehrkräfte mit der technischen und medienpädagogischen Unterstützung sind sowie mit der Unterstützung durch die Schulleitung, welche sich auf die Nutzung digitaler Medien im schulischen Kontext bezieht. Das Antwortformat war sechsstufig und entspricht dem Schulnotenranking von 1 (sehr gut) bis 6 (ungenügend). Für die vorliegende Studie wurden die Items umkodiert, sodass hohe Werte eine hohe Zufriedenheit darstellen. Die Skala weist eine akzeptable interne Konsistenz auf $(\omega=0,74 ; 95 \%$ KI $[0,67 ; 0,78])$.

\subsubsection{Technische Schulausstattung}

Zur Erfassung der technischen Schulausstattung wurde ein Erhebungsinstrument von Breiter et al. (2010) verwendet, welches die Zugangsmöglichkeiten zu sieben digitalen Endgeräten in der Schule und spezifisch für den eigenen Unterricht erfasst. Folgende Endgeräte wurden berücksichtigt: Rechner im Unterrichtsraum, Computerraum, Laptop-Klassensätze, Tablet-Klassensätze, mobile Präsentationseinheiten, Smartboards sowie digitale Kameras, Fotokameras, Aufnahmegeräte. In der vorliegenden Studie wurde die Originalkodierung $(0=$ nicht an der Schule vorhanden; 1 = jederzeit Zugang im Unterricht; 2 =Zugang nur nach Anmeldung und Absprache; $3=$ in unserer Schule nicht vorhanden) angepasst: $0=$ nicht an der Schule vorhanden, $1=$ an der Schule vorhanden. Hieraus wurde ein Summenscore gebildet, welcher eine 
Aussage über die Anzahl beziehungsweise Vielfalt der an der Schule vorhandenen Endgeräte macht (Min/Max: 0/7).

\subsubsection{Wertüberzeugungen zum Einsatz digitaler Medien im Unterricht}

Die Wertüberzeugungen der Lehrkräfte zum Einsatz digitaler Medien im Unterricht wurden anhand von vier Subskalen (Wichtigkeit, Interesse, Nützlichkeit, Kosten) erhoben, die in Anlehnung an Eccles (2005) entwickelt wurden. Eine ähnliche Skala wurde von Rubach und Lazarides (2019) bereits an Lehramtsstudierenden validiert und in der vorliegenden Untersuchung mit geringfügigen Anpassungen für die Befragung von Lehrkräften adaptiert (siehe Rubach und Lazarides 2021a). Jede der vier Subskalen umfasst drei Items mit fünfstufigem Antwortformat von 1=stimme gar nicht zu bis 5= stimme voll und ganz zu. Alle Subskalen weisen eine gute interne Konsistenz auf (Interesse: $\omega=0,90 ; 95 \%$ KI [0,86; 0,93]; Kosten: $\omega=0,71 ; 95 \%$ KI [0,59; 0,77]; Wichtigkeit: $\omega=0,86 ; 95 \%$ KI [0,81; 0,90]; Nützlichkeit: $\omega=0,79$; $95 \%$ KI $[0,73 ; 0,84])$. In der vorliegenden Studie wurden Interesse, Wichtigkeit und Nützlichkeit zu einem Faktor „Wertüberzeugungen“"zusammengefasst (Rubach und Lazarides 2021b), da hier hohe Korrelationen vorliegen. Die interne Konsistenz des latenten Faktors „Wertüberzeugungen“ ist gut $(\omega=0,89 ; 95 \%$ KI $[0,85 ; 0,92])$.

\subsubsection{Digitale Kompetenzselbsteinschätzungen}

Basierend auf dem Instrument von Rubach und Lazarides (2021a) wurde erfasst, wie kompetent Lehrkräfte sich in den grundlegenden (berufsunabhängigen) Kompetenzfacetten zum Umgang mit digitalen Medien einschätzen. Für jeden der sechs Kompetenzbereiche (Suchen und Verarbeiten, Kommunizieren und Kooperieren, Problemlösen und Handeln, Produzieren und Präsentieren, Schützen und sicher Agieren, Analysieren und Reflektieren) wurde ein Item integriert, welches am höchsten auf den jeweiligen Faktor lädt. Zudem wurde in Anlehnung an Siddiq et al. (2016) ein Item integriert, welches einen weiteren Kompetenzbereich, genauer die kompetente Nutzung von Hard- und Software, abbildet. Die insgesamt sieben Items wurden mit fünfstufigem Antwortformat von 1=stimme gar nicht $\mathrm{zu}$ bis $5=$ stimme voll und ganz zu bewertet. Die interne Konsistenz der Skala ist gut $(\omega=0,87 ; 95 \%$ KI $[0,84$; $0,90])$.

\subsubsection{Soziodemografische Merkmale und Strukturmerkmale}

Das Geschlecht der Lehrkräfte wurde binär kodiert ( $0=$ weiblich, $1=$ männlich). Die Berufserfahrung wurde über die Anzahl der Jahre, die die befragte Person bereits als Lehrkraft arbeitet, erfasst. Die Schulform, als relevantes Strukturmerkmal der Schule, wurde über eine dichotomisierte Variable erfasst $(0=$ weitere Schulformen der Sekundarstufe I+ II, 1= Gymnasium). Geschlecht, Berufserfahrung und Schulform wurden als Kovariaten in das Modell integriert. 


\subsection{Statistische Analysen}

Zur Überprüfung der ersten Fragestellung wurde die faktorielle Struktur der von Lehrkräften berichteten Nutzung digitaler Medien zur Strukturierung, kognitiven Aktivierung, konstruktiven Unterstützung und Individualisierung mittels konfirmatorischer Faktorenanalyse (CFA) überprüft. Da unterschiedliche Faktorenladungen der einzelnen Items angenommen werden können, wurde in der CFA ein $\tau$-kongenerisches Modell genutzt. Als Beurteilung der internen Konsistenz der Skalen wurde McDonald's $\omega$ verwendet (Eid und Schmidt 2014).

Zur Untersuchung der indirekten Zusammenhänge zwischen Schulmerkmalen und den Unterrichtsqualitätsdimensionen vermittelt über Lehrkräftemerkmale wurde ein Strukturgleichungsmodell spezifiziert und schrittweise aufgebaut. Im ersten Schritt (Modell 1) wurde der Zusammenhang zwischen Schulmerkmalen, Lehrkräftemerkmalen (als vermittelnder Faktor) und den Unterrichtsqualitätsdimensionen spezifiziert. Im zweiten Schritt (Modell 2) wurden die Kovariaten in das Modell integriert und indirekte Effekte getestet.

Alle Analysen wurden mit Mplus 8.1 (Muthen und Muthen 2018) durchgeführt. Fehlende Werte wurden mittels Full-Information-Maximum-Likelihood-Schätzverfahren geschätzt. Zur Beurteilung der Modellgüte der CFA und der Strukturgleichungsmodelle wurden nach $\mathrm{Hu}$ und Bentler (1999) folgende Gütekriterien überprüft: der RMSEA, der Cooperative-Fit-Index (CFI) und der Tucker-Lewis-Index (TLI) und das SRMR. Die Modellpassung gilt hiernach als gut, wenn der RMSEAWert unter 0,06 liegt, der CFI- und TLI-Wert jeweils über 0,95 sowie das SRMR unter 0,08 .

\section{Ergebnisse}

\subsection{Faktorenstruktur}

Die Ergebnisse der konfirmatorischen Faktorenanalyse (Forschungsfrage 1) verdeutlichen, dass das 4-Faktoren-Modell eine gute Passung $\mathrm{zu}$ den empirischen Daten aufweist: $\chi^{2}(48)=61,80, p=0,09$, RMSEA $=0,03, \mathrm{CFI}=0,99$, TLI $=0,99$, $\mathrm{SRMR}=0,03$. Das in Abb. 2 dargestellte Modell setzt sich aus den Faktoren Strukturierung $(\omega=0,88 ; 95 \% \mathrm{KI}[0,84 ; 0,91])$, Individualisierung $(\omega=0,85 ; 95 \% \mathrm{KI}[0,81$; $0,88])$, kognitive Aktivierung ( $\omega=0,89 ; 95 \% \mathrm{KI}[0,86 ; 0,92])$ und konstruktive Unterstützung $(\omega=0,82 ; 95 \%$ KI $[0,77 ; 0,86])$ zusammen. Alle Faktoren werden mit jeweils drei Items erfasst, deren Faktorladungen in einem Bereich von $\lambda=0,70$ bis $\lambda=0,92$ liegen. Zwischen allen vier Faktoren (Strukturierung, kognitive Aktivierung, konstruktive Unterstützung, Individualisierung) zeigen sich jeweils signifikante mittelstark bis stark positive Zusammenhänge im Bereich $r=0,51$ bis $r=0,76$.

\subsection{Deskriptive Befunde und Skaleninterkorrelationen}

Die deskriptiven Maße und Interkorrelationen der untersuchten Konstrukte sind in Tab. 1 abgebildet. Ergebnisse zur Forschungsfrage 2 zeigen, dass Lehrkräfte im 


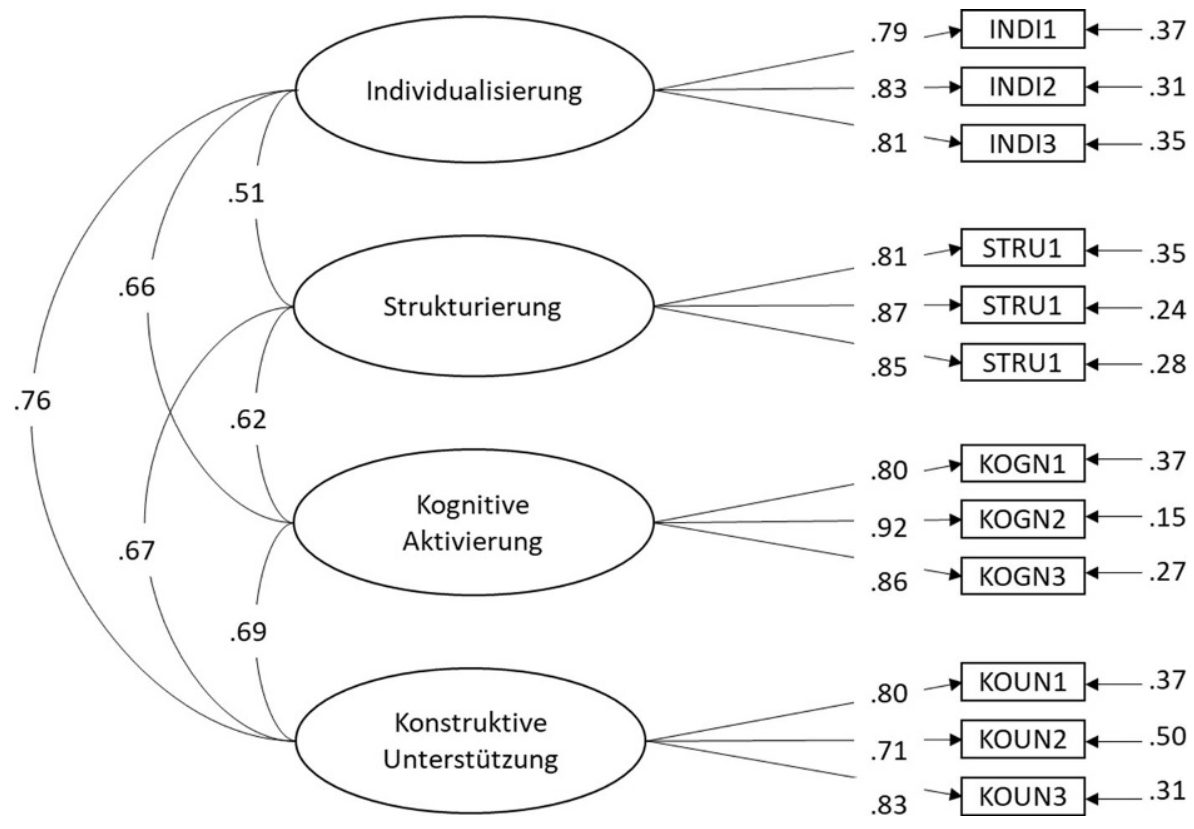

Abb. 2 Vier-faktorielle Struktur der Skala zu den digital gestützten Unterrichtsstrategien. Abgebildet sind Korrelationen, Faktorladungen und Residualvarianzen

Durchschnitt angaben, in einigen Stunden digitale Medien zur Strukturierung ihres Unterrichts zu nutzen $(M=3,37, S D=0,99)$. Die kognitive Aktivierung $(M=2,72$, $S D=0,89)$, Individualisierung $(M=2,68, S D=0,94)$ und konstruktive Unterstützung $(M=2,59, S D=0,91)$ im Unterricht wird mithilfe digitaler Medien in sehr wenigen bis einigen Stunden umgesetzt.

\subsection{Zusammenhänge zwischen den Wertüberzeugungen zum Einsatz digitaler Medien im Unterricht und digital gestützter Unterrichtsqualität}

Zur Beantwortung der Forschungsfragen 3 und 4 wurde ein Strukturgleichungsmodell schrittweise berechnet (Modell 1: ohne Aufnahme der Kovariaten, Modell 2: Aufnahme aller Prädiktoren, siehe Abb. 3). Die Gütekriterien der Modelle verweisen auf eine gute Passung zu den Daten (Modell 1: $\chi^{2}$ (521) 759,94, $p=0,00$; RMSEA $=0,04,90 \%$ KI $[0,03 ; 0,05]$; CFI $=0,95$, TLI=0,95; SRMR $=0,05 ;$ Modell 2: $\chi^{2}(601) 859,05, p=0,00$; RMSEA $=0,04,90 \%$ KI $[0,03 ; 0,05]$; CFI =0,95, $\mathrm{TLI}=0,94$; SRMR=0,05). Beta-Koeffizienten, Standardfehler, Signifikanzniveaus und zugehörige Konfidenzintervalle sind in Tab. 2, 3 und 4 dargestellt. Die Effektstärken der jeweiligen Beta-Koeffizienten sind in Anlehnung an Peterson und Brown (2005) zusammen mit Beta-Koeffizienten und $p$-Werten im Text berichtet.

Die Ergebnisse des finalen Modells zeigen positive Zusammenhänge zwischen Schulmerkmalen, Lehrkräftemerkmalen und Unterrichtsmerkmalen. Die technische Schulausstattung hängt positiv mit der Umsetzung der kognitiven Aktivierung mithilfe digitaler Medien zusammen $(\beta=0,16, p=0,01, r=0,21)$. Die Zufriedenheit mit 


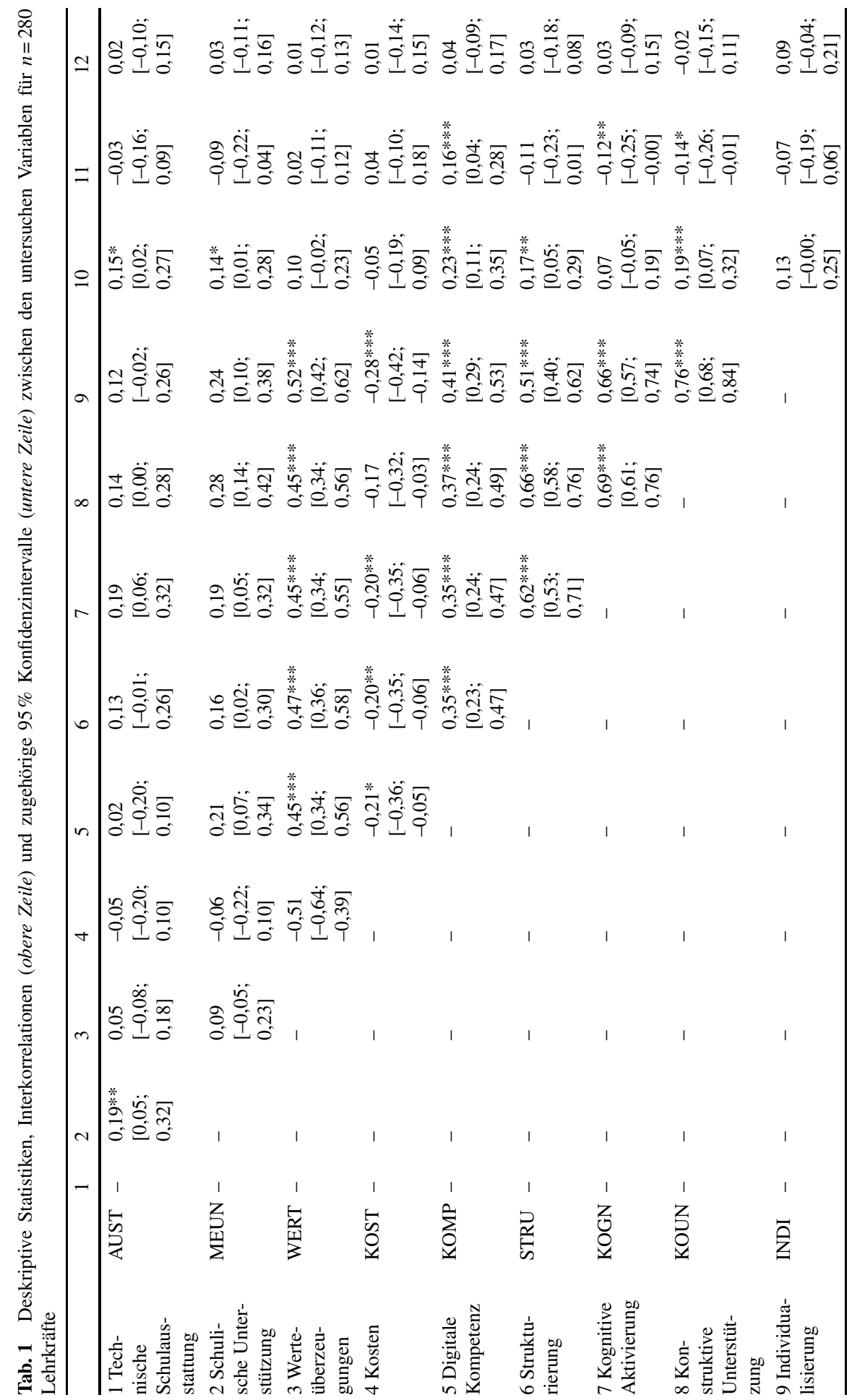




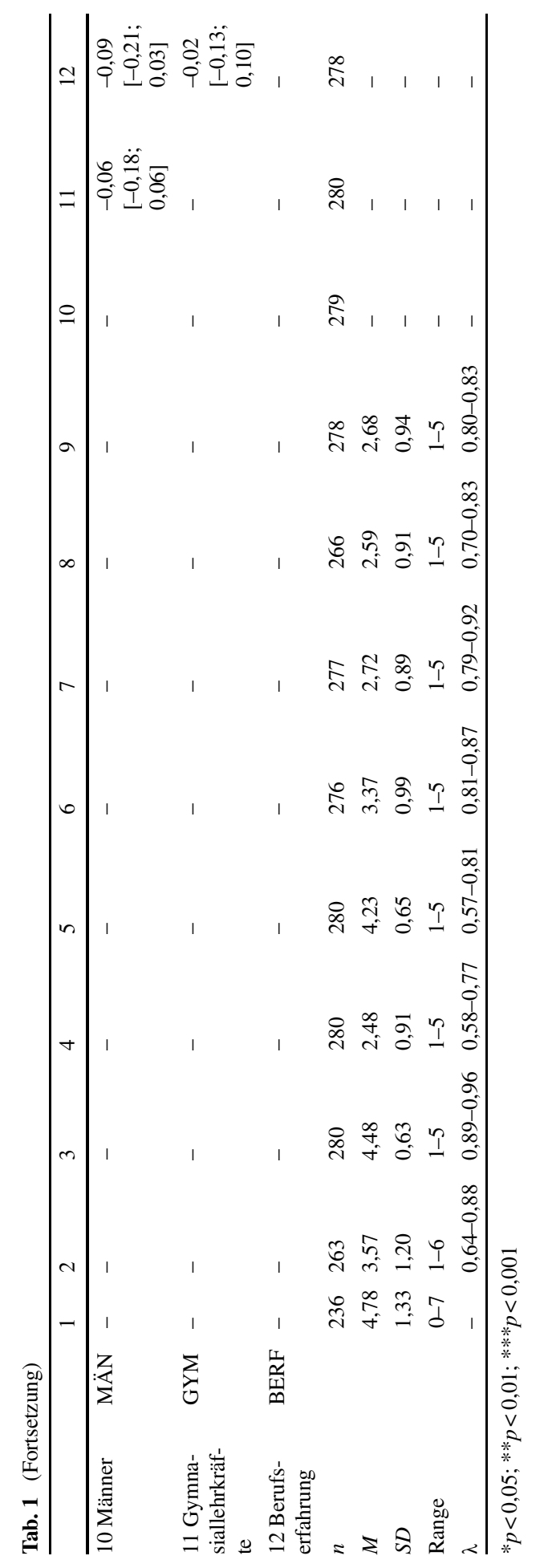




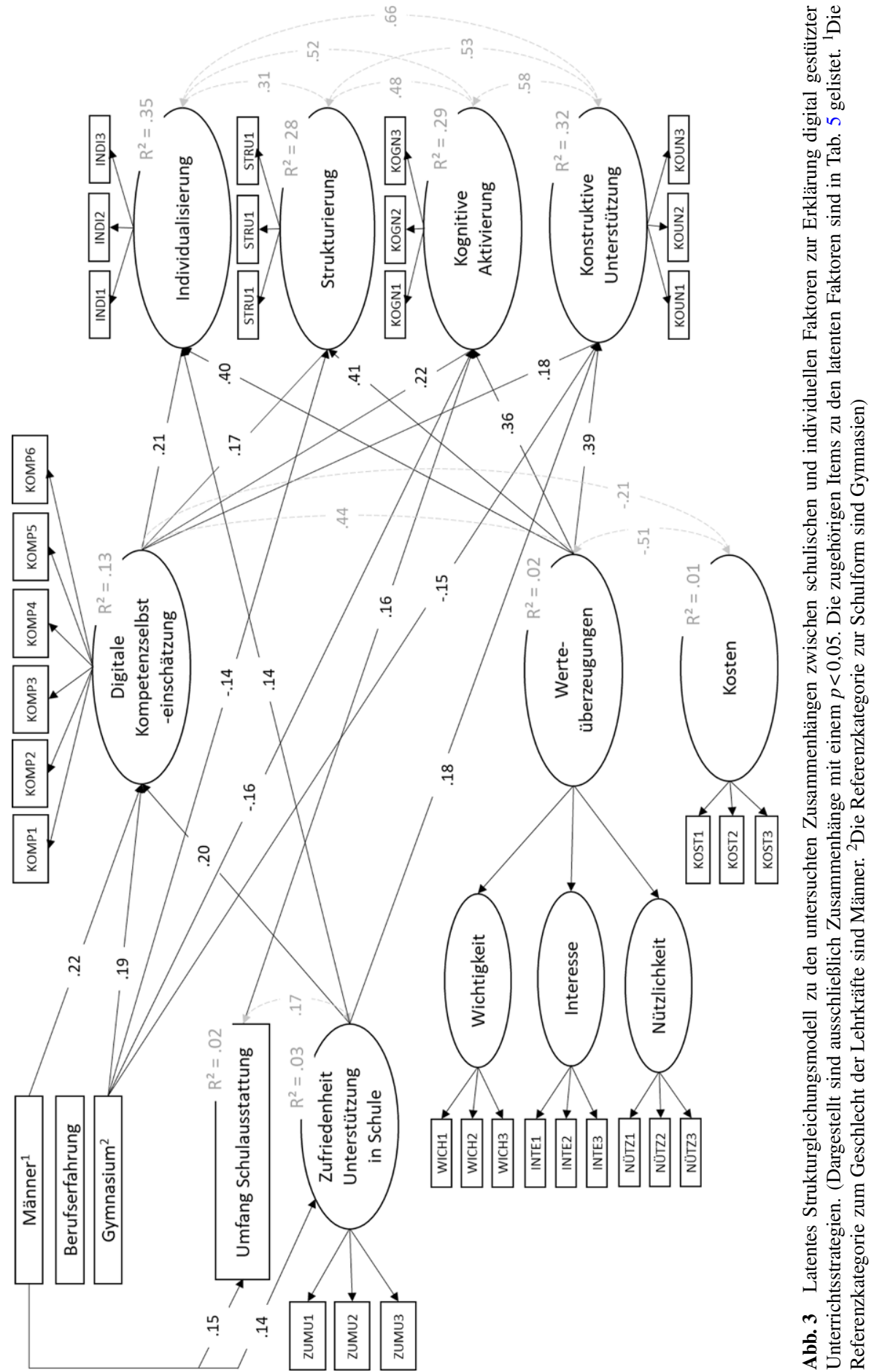




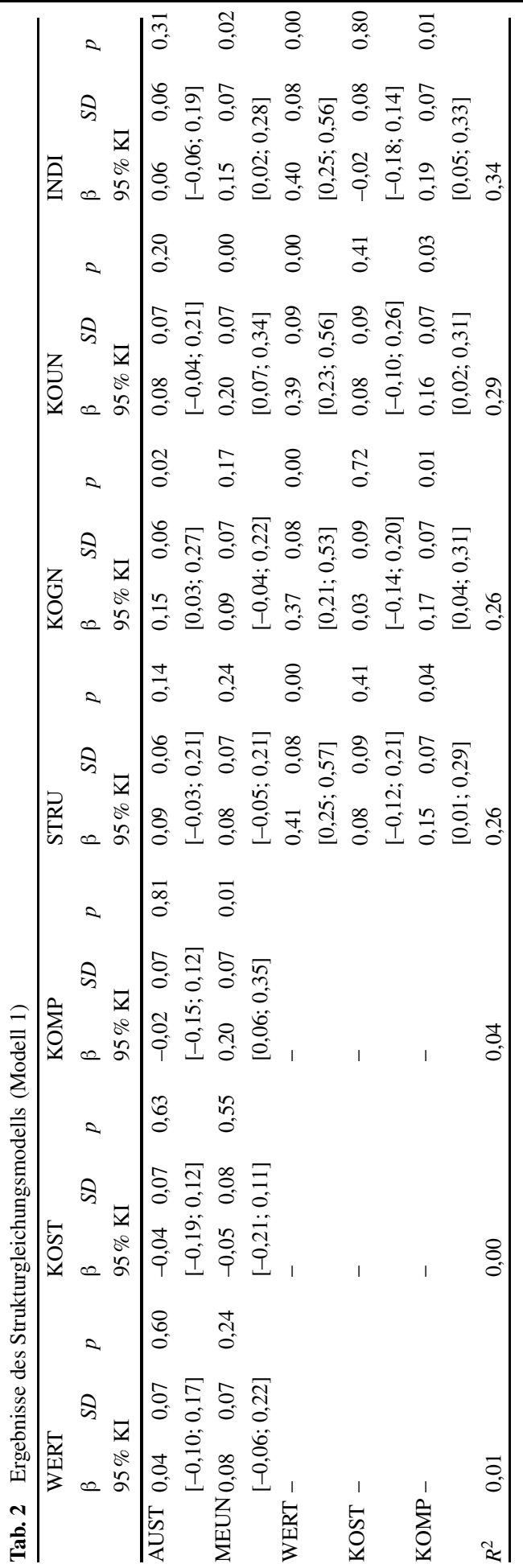




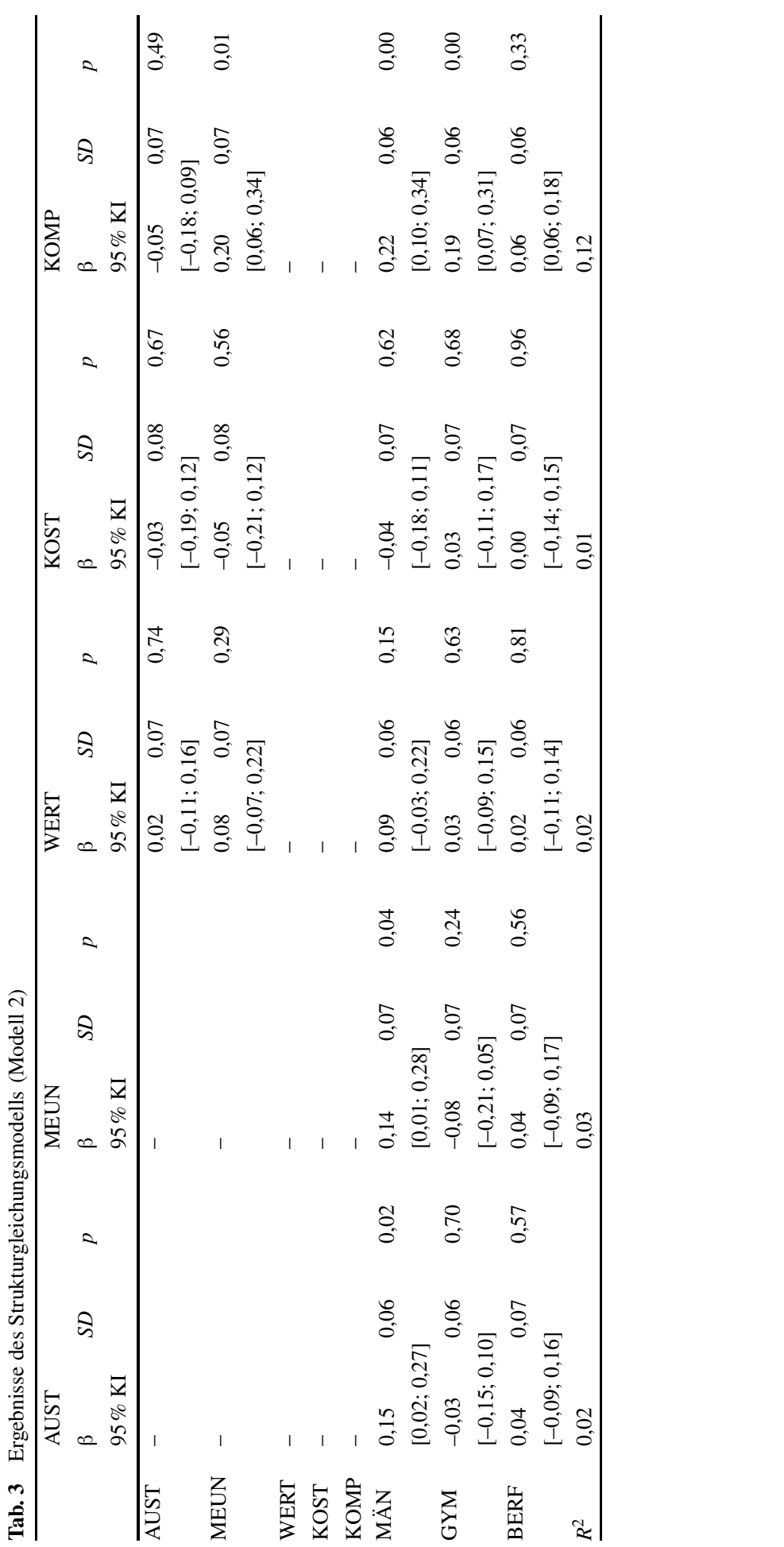




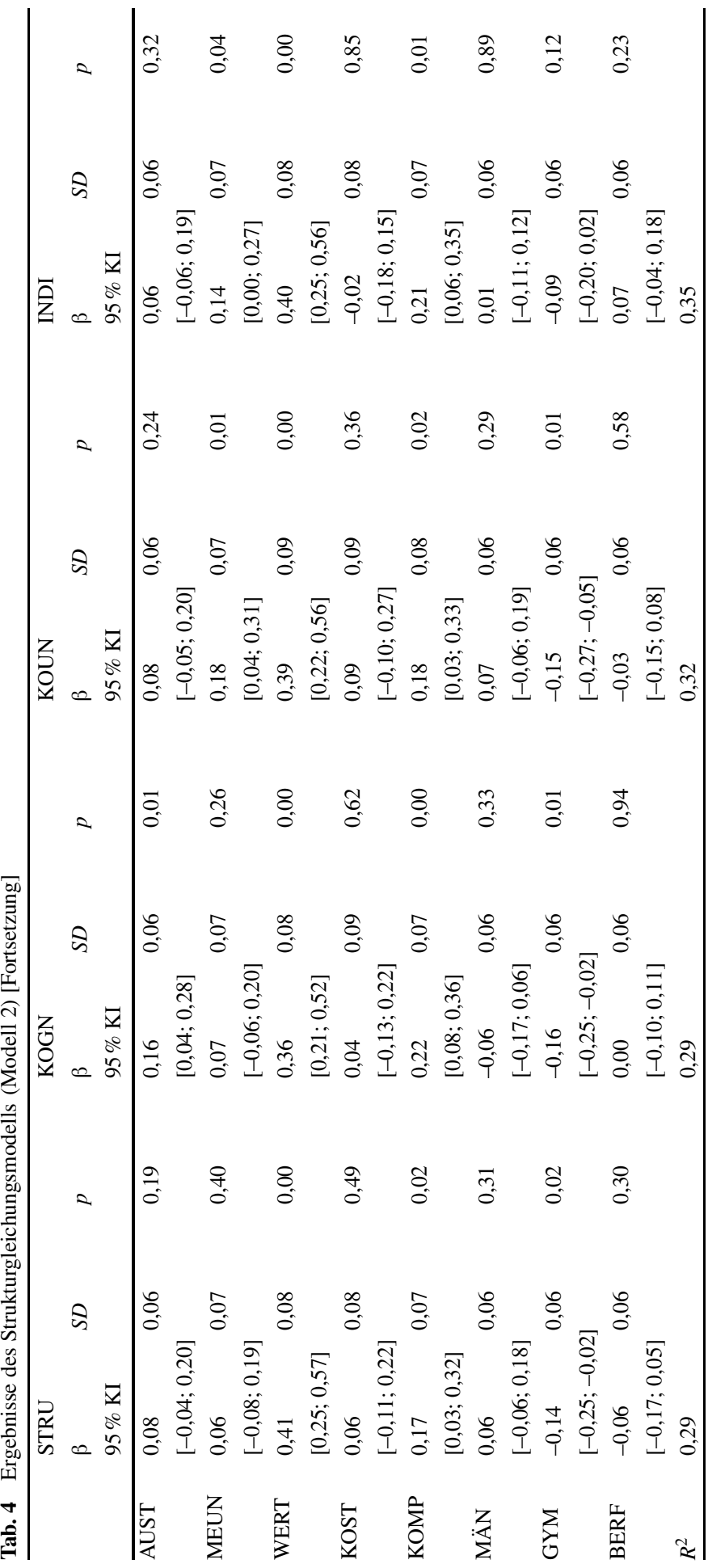


der schulischen Medienunterstützung hängt mit digitalen Kompetenzselbsteinschätzungen $(\beta=0,20, p=0,01, r=0,25)$ sowie mit der selbstberichteten Umsetzung der konstruktiven Unterstützung $(\beta=0,18, p=0,01, r=0,23)$ und Individualisierung zusammen $(\beta=0,14, p=0,04, r=0,19)$. Die von den Lehrkräften berichteten Wertüberzeugungen zum Einsatz digitaler Medien stehen in positivem Zusammenhang mit der Nutzung digitaler Medien zur Umsetzung der kognitiven Aktivierung $(\beta=0,36$, $p<0,001, r=0,41)$, konstruktiven Unterstützung $(\beta=0,39, p<0,001, r=0,44)$, Individualisierung $(\beta=0,40, p<0,001, r=0,45)$ sowie Strukturierung $(\beta=0,41, p<0,001$, $r=0,46)$. Auch die von den Lehrkräften berichteten digitalen Kompetenzselbsteinschätzungen hängen positiv mit der Nutzung digitaler Medien zur Umsetzung der kognitiven Aktivierung $(\beta=0,22, p<0,001, r=0,27)$, konstruktiven Unterstützung $(\beta=0,18, p=0,02, r=0,23)$, Individualisierung $(\beta=0,21, p=0,01, r=0,26)$ sowie Strukturierung zusammen $(\beta=0,17, p<0,001, r=0,22)$. Die Zufriedenheit mit der schulischen Unterstützung bei der Nutzung digitaler Medien wirkt indirekt über die digitale Kompetenzselbsteinschätzung auf die Nutzung digitaler Medien zur Umsetzung der Individualisierung $\left(\beta_{\text {ind }}=0,04, \mathrm{SE}=0,02, p=0,05,95 \% \mathrm{KI}[0,00 ; 0,08]\right.$, $r=0,09)$ und kognitiven Aktivierung $\left(\beta_{\text {ind }}=0,04, \mathrm{SE}=0,02, p=0,04,95 \% \mathrm{KI}[0,00\right.$; 0,08], $r=0,09)$.

Lehrer schätzen sich im Vergleich zu Lehrerinnen als digital kompetenter ein $(\beta=0,22, p<0,001, r=0,27)$. Gymnasiallehrkräfte schätzen sich im Vergleich zu Lehrkräften, die an anderen Schularten der Sekundarstufe unterrichten, ebenfalls als digital kompetenter ein $(\beta=0,19, p<0,001, r=0,24)$. Die technische Schulausstattung $(\beta=0,15, p=0,02, r=0,20)$ sowie die Zufriedenheit mit der schulischen Unterstützung bei der Nutzung digitaler Medien $(\beta=0,14, p=0,04$, $r=0,19)$ sind positiv mit dem Geschlecht der Lehrkräfte assoziiert. Zudem berichten Gymnasiallehrkräfte im Vergleich zu Lehrkräften anderer Schularten der Sekundarstufe seltener, dass digitale Medien zur Umsetzung von kognitiver Aktivierung $(\beta=-0,16, p=0,01, \quad r=-0,21)$, Strukturierung $(\beta=-0,14$, $p=0,02, r=-0,19)$ und konstruktiver Unterstützung $(\beta=-0,15, p=0,01, r=-0,20)$ eingesetzt werden. Zusätzliche Analysen zeigen auf, dass diese negativen Effekte durch digitale Kompetenzselbsteinschätzungen erklärt werden (Gymnasi$\mathrm{um} \rightarrow$ Kompetenzselbsteinschätzungen $\rightarrow$ Individualisierung: $\beta_{\text {ind }}=0,04, S E=0,02$, $p=0,04, \quad 95 \%$ KI $[0,00 ; 0,08], \quad r=0,09), \quad$ Gymnasium $\rightarrow$ Kompetenzselbsteinschätzungen $\rightarrow$ kognitive Aktivierung $\left(\beta_{\text {ind }}=0,04, S E=0,02, p=0,03,95 \% \mathrm{KI}\right.$ $[0,00 ; 0,08], r=0,09)$.

\section{Diskussion}

Eine aktuelle Herausforderung der Bildungsforschung ist die systematische Analyse der Schnittstellen zwischen Forschung zu Tiefenstrukturen des Unterrichts und Forschung zur Veränderung von Unterrichtsprozessen durch digitale Medien. Ziel der vorliegenden Studie ist daher die empirische Untersuchung der Nutzung digitaler Medien zur Gestaltung qualitätsvoller Unterrichtsprozesse. Unsere Annahmen bestätigend (Hypothese 1) konnte die angenommene Faktorenstruktur aufgezeigt werden - die angenommenen Unterrichtsdimensionen kognitive Aktivierung, kon- 
struktive Unterstützung, Strukturierung sowie Individualisierung wurden anhand der vorliegenden Daten identifiziert. Die Ergebnisse der vorliegenden Studie verdeutlichen zudem wie angenommen (Hypothese 2), dass Lehrkräfte digitale Medien zur Strukturierung von Unterrichtsprozessen nutzen sowie diese implementieren, um Prozesse der kognitiven Aktivierung, konstruktiven Unterstützung und Individualisierung umzusetzen. Jedoch zeigte sich nicht, dass digitale Medien weniger häufig genutzt werden, um Unterrichtsprozesse kognitiv aktivierend zu gestalten.

Die Ergebnisse der Studie verdeutlichen des Weiteren, dass die grundlegenden (berufsunabhängigen) digitalen Kompetenzselbsteinschätzungen den Zusammenhang zwischen der Zufriedenheit mit der schulischen Unterstützung zur Nutzung digitaler Medien und der Umsetzung der kognitiven Aktivierung und Individualisierung mithilfe digitaler Medien vermitteln.

\subsection{Schulmerkmale und der Einsatz digitaler Medien}

Die Befunde der Studie verweisen darauf, dass Lehrkräfte höhere digitale Kompetenzselbsteinschätzungen berichten, wenn sie zufriedener mit der schulischen Unterstützung bei der Nutzung digitaler Medien sind. Diese Befunde zeigen sich auch in der Analyse von Sundqvist et al. (2021). Überraschend ist, dass eine zufriedenstellende schulische Medienunterstützung nicht mit geringeren wahrgenommenen Kosten (z.B. Zeitaufwand) zusammenhängt. Die technische Schulausstattung war positiv mit der kognitiven Aktivierung assoziiert. Diese Ergebnisse bestätigen vorherige Befunde von Lucas et al. (2021), die verdeutlichen, dass die Anzahl der in der Schule zur Verfügung stehenden digitalen Geräte mit der Nutzung digitaler Medien in Unterrichtsprozessen korrelieren. Für die konstruktive Unterstützung, Strukturierung und Individualisierung mit digitalen Medien zeigte die technische Schulausstattung hingegen keine Relevanz. Dieses Ergebnis könnte darauf hinweisen, dass nur für die Umsetzung bestimmter Unterrichtsaktivitäten eine vielfältige technische Schulausstattung benötigt wird, beispielsweise wenn Lernende digitale Medien nutzen sollen (so wie in dem Messinstrument zur kognitiven Aktivierung erfasst).

\subsection{Individuelle Überzeugungen der Lehrkräfte und der Einsatz digitaler Medien}

Auch unsere Annahmen zu der Bedeutung individueller Überzeugungen (Hypothese 3) für den Einsatz digitaler Medien konnte bestätigt werden. Die Befunde entsprechen theoretischen Annahmen des Erwartungs-Wert-Modells (Eccles et al. 1983). Aufgezeigt wird, dass Wertüberzeugungen und digitale (berufsunabhängige) Kompetenzselbsteinschätzungen bedeutsam für das berufsbezogene Nutzungsverhalten digitaler Medien von Lehrkräften sind (Backfisch et al. 2021; Petko 2012). Daraus lässt sich einerseits ableiten, dass Lehrkräfte sich digital kompetent wahrnehmen müssen und anderseits schlussfolgern, dass Lehrkräfte den Wert zum Einsatz digitaler Medien erkennen müssen, damit sie digitale Medien zur Umsetzung qualitätsvoller Unterrichtsprozesse einsetzen. 
Entgegen unserer Annahmen (Hypothese 4) konnte in der vorliegenden Studie nicht bestätigt werden, dass die wahrgenommenen Kosten die Umsetzung der Unterrichtsqualitätsdimensionen mithilfe digitaler Medien hemmen. Damit können Annahmen des Erwartungs-Wert-Modells (Eccles et al. 1983) nicht bestätigt werden, dass Individuen insbesondere dann Handlungen ausführen, wenn diese mit niedrig empfundenen (psychologischen) Kosten verbunden sind. Eine mögliche Erklärung für diese Befunde ist, dass sich in der vorliegenden Stichprobe nur eine sehr geringe Wahrnehmung psychologischer Kosten in Bezug auf digitale Medien zeigte $(M=2,48, S D=0,91)$. Andererseits könnte die berufliche Verpflichtung von Relevanz sein - auch wenn Lehrkräfte mit dem Einsatz digitaler Medien Kosten verbinden, könnten sie es als wichtige berufliche Verantwortung wahrnehmen, Lernenden die Möglichkeit zu geben, mit, von und über Medien zu lernen und daher unabhängig der wahrgenommenen psychologischen Kosten digitale Medien in Unterrichtsprozessen einsetzen.

Bestätigend zu Hypothese 5 verweisen unsere Ergebnisse darauf, dass die Zufriedenheit mit der schulischen Unterstützung zur Nutzung digitaler Medien vermittelt über die digitale (berufsunabhängige) Kompetenzselbsteinschätzungen mit der Umsetzung der Unterrichtsqualitätsdimensionen durch die Nutzung digitaler Medien zusammenhängt. Damit kann die Annahme des Erwartungs-Wert-Modells von Eccles et al. (1983) dahingehend bestätigt werden, dass Umweltfaktoren (Schulmerkmale) vermittelt über motivationale Überzeugungen (Lehrerüberzeugungen) auf Verhaltensweisen (Unterrichtspraktiken) wirken. Jedoch sollte an dieser Stelle darauf hingewiesen werden, dass die Befunde dieser Studie querschnittlich sind. Interessant ist zudem, dass Sundqvist et al. (2021) einen negativen Zusammenhang zwischen der Zufriedenheit zur schulischen Unterstützung und der Umsetzung der Unterrichtsqualitätsdimensionen mithilfe digitaler Medien demonstrierten. Die Autorengruppe diskutierte diesbezüglich, inwiefern Lehrkräfte, welche die Unterstützung durch die Schule bei der Nutzung digitaler Medien in Anspruch nehmen, weniger digital kompetent sind und daher mehr Unterstützung benötigen.

\subsection{Die Bedeutung soziodemografischer Merkmale der Lehrkräfte und struktureller Merkmale der Schule}

Unsere Annahme zur Bedeutung des Geschlechts (Hypothese 6) konnte nur teilweise bestätigt werden. In unserer Studie fanden wir keinen signifikanten Zusammenhang zwischen dem Geschlecht der Lehrkräfte und der Nutzung digitaler Medien zur Umsetzung der Unterrichtsqualität. Demnach können die Ergebnisse von Drossel et al. (2017) und Tezci (2010), die einen Zusammenhang zwischen Geschlecht und Nutzungshäufigkeit digitaler Medien im Unterricht nahelegen, nicht für die Nutzung digitaler Medien zur Umsetzung der Unterrichtsprozessqualität repliziert werden. Auch Lucas et al. (2021) zeigten, dass Lehrer im Vergleich zu Lehrerinnen häufiger digitale Medien zur Prüfungsgestaltung und -abnahme sowie insgesamt zur methodischen und pädagogischen Unterrichtsgestaltung einsetzen. Angesichts unserer Befunde stellt sich für zukünftige Studien die Frage, inwiefern die geschlechtsspezifische Mediensozialisation eine Relevanz für die Häufigkeit, nicht aber für die Art der Nutzung digitaler Medien innehat. 
Unsere Annahmen bestätigend (Hypothese 6) konnten bisherige Befunde (Rohatgi et al. 2016; Scherer et al. 2017) repliziert werden, da Lehrer im Vergleich zu Lehrerinnen höhere grundlegende (berufsunabhängige) digitale Kompetenzselbsteinschätzungen berichteten. Die Frage, die sich anschließend stellt, ist, welche Faktoren Lehrerinnen darin bestärken, digitale Medien einzusetzen, wenn sie ihre eigenen Fähigkeiten zum kompetenten Umgang mit digitalen Medien im Vergleich zu männlichen Lehrkräften jedoch als weniger ausgeprägt wahrnehmen.

Wichtig zu diskutieren ist der aufgezeigte Zusammenhang zwischen Geschlecht und technischer Schulausstattung. Angenommen werden könnte, dass der Zusammenhang zwischen Schulausstattung und Geschlecht nur dann signifikant ist, wenn Lehrkräfte ein STEM-Fach unterrichten. In unserer Stichprobe berichten Lehrer signifikant häufiger davon, Informatik $\left(\chi^{2}(1)=6,04, p=0,01\right)$, Physik $\left(\chi^{2}(1)=4,86\right.$, $p=0,03)$, Sport $\left(\chi^{2}(1)=7,86, p=0,00\right)$ und Arbeit-Wirtschaft-Technik $\left(\chi^{2}(1)=7,86\right.$, $p=0,01)$ zu unterrichten. Frauen in dieser Stichprobe hingegen geben proportional häufiger an, dass sie Englisch $\left(\chi^{2}(1)=13,10, p=0,00\right)$, Französisch $\left(\chi^{2}(1)=10,43\right.$, $p=0,00)$ und Kunst $\left(\chi^{2}(1)=6,59, p=0,01\right)$ unterrichten. Zusätzliche Analysen machen deutlich, dass der Zusammenhang zwischen Schulausstattung und Geschlecht nur dann signifikant ist, wenn Lehrkräfte ein STEM-Fach unterrichten $(\beta=0,83$, $\mathrm{SE}=0,35,90 \%$ KI $[0,24 ; 1,12], p=0,02$; Simple Slope $(\mathrm{STEM}): \beta_{\mathrm{STEM}}=0,68$, S.E. $=0,22 ; p=0,00,90 \%$ KI $[0,24 ; 1,12]$, Simple Slope (kein STEM): $\beta_{\text {NOSTEM }}=-16$, S.E. $=0,27 ; p=0,57,90 \%$ KI $[-0,69 ; 0,38])$. Angenommen werden könnte, dass Lehrkräfte in STEM-Fächern häufiger Medien benötigen und auch nutzen, um zu unterrichten.

Die Annahme, dass die Berufserfahrung negativ mit der Nutzung digitaler Medien zur Umsetzung der Unterrichtsqualitätsdimensionen korreliert (Hypothese 7), konnte nicht bestätigt werden. Die vorliegende Untersuchung kann damit den negativen Zusammenhang zwischen Berufserfahrung und der Häufigkeit der Nutzung digitaler Medien im Unterricht aus früheren Studien (siehe Gil-Flores et al. 2017; Tezci 2010) nicht bestätigen.

Hypothese 8 konnte bestätigt werden - Gymnasiallehrkräfte gaben im Vergleich zu Lehrkräften anderer Sekundarschulformen an, weniger häufig digitale Medien zur Umsetzung der Unterrichtsqualität zu nutzen. Interessanterweise zeigen Befunde, dass digitale Medien an Gymnasien insgesamt häufiger eingesetzt werden (Schmid et al. 2017; Wetterich et al. 2014). Jedoch können Befunde der ICILS-Studie 2018 (Schaumburg et al. 2019) durch die vorliegende Studie bestätigt werden, die darauf verweisen, dass Lernende am Gymnasium weniger häufig berichten, digitale Medien in der Schule für schulbezogene Zwecke zu nutzen. Auch mit Daten von Lehrkräften in dieser Studie zeigt sich, dass Gymnasiallehrkräfte tendenziell weniger häufig digitale Medien zur Strukturierung, kognitiven Aktivierung und konstruktiven Unterstützung nutzen.

\subsection{Limitationen und Ausblick}

Eine Limitation der Studie ist, dass Lehrkräfte aus dem Bereich Informatik in der Stichprobe überproportional vertreten sind. Der fachliche Hintergrund der Lehrkräfte könnte mit einer hohen Affinität zum Einsatz digitaler Medien im Unterricht 
verbunden sein. Nachfolgende Untersuchungen, die Populationen von Lehrkräften aus verschiedenen Fachbereichen einbeziehen, sind notwendig. Insgesamt sollten die Ergebnisse unserer Studie in weiterführenden Studien anhand weiterer Stichproben validiert werden. Nochmals wird darauf verwiesen, dass durch die Art der Erhebung (Onlinebefragung über Twitter und Fobizz) eine spezifische Gruppe von Lehrkräften angesprochen wurde, die häufiger digitale Medien nutzen könnte. Zudem sind in unserer Stichprobe im Vergleich zur Gesamtverteilung von Lehrkräften (Statistisches Bundesamt 2019) proportional mehr männliche Lehrkräfte vertreten. Des Weiteren beziehen unsere Analysen nur Lehrkräfte ein, die an Schulen der Sekundarstufe tätig sind. Wünschenswert wäre es, die aufgezeigten Zusammenhänge im Vergleich von Grund- und Sekundarschullehrkräften sowie von Lehrerinnen und Lehrern anhand einer repräsentativeren Stichprobe zu prüfen. Trotz der Spezifität der vorliegenden Stichprobe konnten Befunde aus internationalen Studien (Sundqvist et al. 2021; Lucas et al. 2021) repliziert werden. Unsere Befunde verweisen auf die Notwendigkeit einer zufriedenstellenden Unterstützung durch die Schule, hoher digitaler (berufsunabhängiger) Kompetenzselbsteinschätzung und positiver Wertüberzeugungen der Lehrkräfte bezüglich des Einsatzes digitaler Medien für eine qualitätsvolle Umsetzung des Unterrichts durch die Nutzung digitaler Medien.

In der hier vorliegenden Studie wurden Lehrkräfte gefragt, inwiefern digitale Medien zur Umsetzung von Unterrichtsqualitätsdimensionen genutzt werden (Klieme 2019). Jedoch können wir keine Aussagen darüber treffen, inwieweit die Nutzung digitaler Medien in diesem Zusammenhang zu einer effektiveren Förderung der Bildungsentwicklung von Lernenden beiträgt. Zudem wurden die Unterrichtsqualitätsdimensionen über Selbstberichte erfasst, sodass die in der Untersuchung gefundenen Zusammenhänge auf einen common method bias zurückführbar sein können (Siemsen et al. 2010). Gegen diese Annahme spricht jedoch, dass nicht alle subjektiven Überzeugungen von gleicher Relevanz für die Nutzung digitaler Medien zur Umsetzung der Unterrichtsqualitätsdimensionen sind.

\subsection{Wissenschaftliche und praktische Implikationen}

Zusammenfassend ermöglicht die vorliegende Studie einen differenzierten Blick auf die Nutzung digitaler Medien zur Umsetzung verschiedener Unterrichtsqualitätsdimensionen. Es konnte ein reliables Instrument für die theoriegeleitete Erfassung der Nutzung digitaler Medien zur Umsetzung der Unterrichtsqualitätsdimensionen vorgelegt werden - damit ist eine differenzierte Analyse der unterrichtlichen Nutzung digitaler Medien ermöglicht.

Für die zukünftige Forschung zeigt sich die Notwendigkeit weiterführender Untersuchungen zu den Wirkzusammenhängen zwischen Schulmerkmalen, Lehrkräftemerkmalen und der Nutzung digitaler Medien zur Umsetzung qualitätsvoller Unterrichtsprozesse. Da unsere Studie querschnittliche Zusammenhangsmuster untersucht hat, sollten die identifizierten Zusammenhänge in zukünftigen längsschnittlichen Studien weiterführend untersucht werden. Zudem wäre es in zukünftigen Studien sinnvoll, auch mittels Videoanalysen die Implikationen des Einsatzes digitaler Medien für die Prozessqualität von Unterricht zu untersuchen. Weiterhin stellt sich die Frage, welche Wissenskomponenten von Lehrkräften dazu beitragen, Unterricht mit 
digitalen Medien qualitätsvoll zu gestalten. Beispielsweise könnte hier untersucht werden, welche Bedeutung die TPCK-Komponenten für solche Unterrichtsprozesse innehaben (Guggemos und Seufert 2021). Erste Befunde legen nahe, dass die Selbsteinschätzungen zum technisch-pädagogischen Wissen (TPK) auf die Nutzung digitaler Medien im Unterricht wirken, während die Selbsteinschätzungen zum technisch-pädagogischen Inhaltswissens (TPCK) aufklären, wie Lehrkräfte die Nutzung digitaler Medien als eigenständigen Unterrichtsgegenstand unterrichten (Guggemos und Seufert 2021).

Unsere Befunde sind zudem relevant für die schulische Praxis. Aufgezeigt wird, dass eine vielfältige technische Schulausstattung allein nicht ausreicht, damit Lehrkräfte digitale Medien zur Gestaltung qualitätsvoller Lernprozesse einsetzen. Unsere Befunde verweisen darauf, dass Lehrkräfte in ihrem Unterrichtshandeln generell davon profitieren könnten, wenn ihnen durch die Schule selbst eine technische und medienpädagogische Unterstützung angeboten wird (siehe auch Ertmer et al. 2012). Weiterhin weisen unsere Befunde darauf hin, dass Lehrkräfte digitale Medien zur Umsetzung qualitätsvoller Unterrichtsprozesse dann nutzen, wenn diese den Einsatz der digitalen Medien im Unterricht als wertvoll wahrnehmen und sich digital kompetent in grundlegenden (berufsunabhängigen) Kompetenzbereichen einschätzen. Diese Überlegungen sollten in die Planung und Gestaltung zukünftiger Fortund Weiterbildungen Eingang finden. Um digitale Kompetenzeinschätzungen zu stärken, sollten eigene Nutzungserfahrungen mit digitalen Medien für die Gestaltung von Unterrichtsprozessen ein Element der Lehrkräfteaus- und -fortbildung sein (siehe Rubach et al. 2019). 


\section{Anhang}

Tab. 5 Itemwortlaut und Faktorenladungen der latent modellierten Faktoren aus dem finalen Modell 2 (siehe Abb. 3)

\begin{tabular}{lll}
\hline Item $\quad$ Itemwortlaut & $\lambda$ \\
\hline
\end{tabular}

\section{A Schulmerkmale}

A1 Zufriedenheit mit der schulischen Medienunterstützung

(Bitte bewerten Sie die folgenden Rahmenbedingungen in Ihrer Schule mit Schulnoten.)

$\begin{array}{llr}\text { ZUMU1 } & \text { Technischer Support } & 0,63\end{array}$

$\begin{array}{ll}\text { ZUMU2 Medienpädagogische Unterstützung } & 0,89\end{array}$

ZUMU3 Unterstützung durch die Schulleitung (zum Einsatz digitaler Medien) $\quad 0,56$

\section{B Lehrkräftemerkmale}

B1 Interesse

INTE1 Es freut mich, wenn ich SchülerInnen bei schulischen Angelegenheiten unter- $\quad 0,77$

INTE2 Der Einsatz digitaler Medien im Unterricht macht mir Spaß

INTE3 Der Einsatz digitaler Medien im Unterricht ist interessant

B2 Wichtigkeit

WICH1 Es ist mir wichtig, dass ich als Lehrkraft digitale Medien im Unterricht einsetze

WICH2 Der Einsatz digitaler Medien im Unterricht ist ein wichtiger Teil in meiner

WICH3 Es ist für mich selbstverständlich, dass ich digitale Medien im Unterricht ein-

B3 Nützlichkeit

NÜTZ1 Ich finde den Einsatz digitaler Medien im Unterricht nützlich, um SchülerInnen in der schulischen Entwicklung zu unterstützen

NÜTZ2 Ich bin der Meinung, dass ich SchülerInnen in schulischen Fragen besser fördern kann, wenn ich digitale Medien im Unterricht einsetze

NÜTZ3 Der Einsatz digitaler Medien im Unterricht hat für Lehrkräfte Vorteile

B4 Werteüberzeugungen zum Einsatz digitaler Medien (Second-Order-Faktor aus B1, B2 und B3)

Interesse B1

Nützlichkeit B2 -

Wichtigkeit B3 -

B5 Kosten

KOST1

Der Einsatz digitaler Medien im Unterricht kostet mich Zeit und Energie

KOST2

Es kostet mich Überwindung, digitale Medien im Unterricht einzusetzen 
Tab. 5 (Fortsetzung)

\begin{tabular}{|c|c|c|}
\hline Item & Itemwortlaut & $\lambda$ \\
\hline \multicolumn{3}{|c|}{ B6 Digitale Kompetenzselbsteinschätzungen } \\
\hline KOMP1 & $\begin{array}{l}\text { Ich kann Informationen, Informationsquellen und Daten im digitalen Raum } \\
\text { kritisch bewerten }\end{array}$ & 0,57 \\
\hline KOMP2 & $\begin{array}{l}\text { Ich kann Verhaltensregeln bei digitalen Interaktionen und Kooperationen an- } \\
\text { wenden }\end{array}$ & 0,60 \\
\hline KOMP3 & Ich kann digitale Inhalte in verschiedenen Formaten präsentieren & 0,75 \\
\hline KOMP4 & $\begin{array}{l}\text { Ich kenne die Gefahren und Risiken in digitalen Umgebungen und berücksich- } \\
\text { tige diese }\end{array}$ & 0,71 \\
\hline KOMP5 & Ich kann digitale Werkzeuge, Tools und Plattformen bedarfsgerecht einsetzen & 0,79 \\
\hline KOMP6 & Ich kenne Funktionsweisen und grundlegende Prinzipien des digitalen Raumes & 0,81 \\
\hline KOMP7 & Ich kann die Wirkung von Medien im digitalen Raum analysieren & 0,61 \\
\hline
\end{tabular}

\section{Unterrichtsmerkmale}

\section{C1 Individualisierung}

INDI1 Mithilfe digitaler Medien gebe ich schnellen SchülerInnen Extraaufgaben

INDI2 Mithilfe digitaler Medien variiere ich die Aufgabenstellungen, um Schüler-

INDI3 Wenn SchülerInnen etwas nicht verstanden haben, vergebe ich gezielte Zusatzaufgaben mithilfe digitaler Medien

C2 Strukturierung

STRU1 Mithilfe digitaler Medien mache ich deutlich, was meine SchülerInnen sich merken sollen

STRU2 Im Unterricht fasse ich mithilfe digitaler Medien das Wichtigste nochmals zusammen

STRU3 Im Unterricht halten wir mithilfe digitaler Medien Rückblick auf das, was wichtig ist

C3 Kognitive Aktivierung

KOGN1 In meinem Unterricht bitte ich SchülerInnen darum, verschiedene Wege zur Lösung eines Problems mithilfe digitaler Medien aufzuzeigen

KOGN2 In meinem Unterricht bitte ich darum, dass die SchülerInnen ihre Gedankengänge mithilfe digitaler Medien gründlich erklären

KOGN3 In meinem Unterricht erwarte ich oft von den SchülerInnen, dass sie ihre Arbeit mithilfe digitaler Medien im Detail erklären

C4 Konstruktivistische Unterstützung

KOUN1 Im Unterricht informiere ich durch den Einsatz von digitalen Medien die SchülerInnen über deren Fortschritte

KOUN2 Im Unterricht bespreche ich mithilfe digitaler Medien schwierige Aufgaben mit den SchülerInnen

KOUN3 Durch den Einsatz von digitalen Medien sage ich den SchülerInnen, was diese verbessern können

Funding Open Access funding enabled and organized by Projekt DEAL.

Open Access Dieser Artikel wird unter der Creative Commons Namensnennung 4.0 International Lizenz veröffentlicht, welche die Nutzung, Vervielfältigung, Bearbeitung, Verbreitung und Wiedergabe in jeglichem Medium und Format erlaubt, sofern Sie den/die ursprünglichen Autor(en) und die Quelle ordnungsgemäß nennen, einen Link zur Creative Commons Lizenz beifügen und angeben, ob Änderungen vorgenommen wurden. 
Die in diesem Artikel enthaltenen Bilder und sonstiges Drittmaterial unterliegen ebenfalls der genannten Creative Commons Lizenz, sofern sich aus der Abbildungslegende nichts anderes ergibt. Sofern das betreffende Material nicht unter der genannten Creative Commons Lizenz steht und die betreffende Handlung nicht nach gesetzlichen Vorschriften erlaubt ist, ist für die oben aufgeführten Weiterverwendungen des Materials die Einwilligung des jeweiligen Rechteinhabers einzuholen.

Weitere Details zur Lizenz entnehmen Sie bitte der Lizenzinformation auf http://creativecommons.org/ licenses/by/4.0/deed.de.

\section{Literatur}

Akçayır, M., \& Akçayır, G. (2017). Advantages and challenges associated with augmented reality for education: a systematic review of the literature. Educational Research Review, 20, 1-11. https://doi. org/10.1016/j.edurev.2016.11.002.

Amhag, L., Hellström, L., \& Stigmar, M. (2019). Teacher educators' use of digital tools and needs for digital competence in higher education. Journal of Digital Learning in Teacher Education, 35(4), 203-220. https://doi.org/10.1080/21532974.2019.1646169.

Aslan, A. \& Zhu, C. (2016). Influencing factors and integration of ICT into teaching practices of preservice and starting teachers. International Journal of Research in Education and Science (IJRES), 2(2), 359-370.

Backfisch, I., Lachner, A., Stürmer, K., \& Scheiter, K. (2021). Variability of teachers' technology integration in the classroom: a matter of utility! Computers \& Education, 166, 104159. https://doi.org/10. 1016/j.compedu.2021.104159.

Baumert, J., Kunter, M., Brunner, M., Krauss, S., Blum, W., \& Neubrand, M. (2004). Mathematikunterricht aus Sicht der PISA-Schülerinnen und -Schüler und ihrer Lehrkräfte. In M. Prenzel (Hrsg.), PISA 2003. Der Bildungsstand der Jugendlichen in Deutschland ; Ergebnisse des zweiten internationalen Vergleichs (S. 314-354). Münster: Waxmann.

Breiter, A., Welling, S., \& Stolpmann, B.E. (2010). Medienkompetenz in der Schule. Integration von Medien in den weiterführenden Schulen in Nordrhein-Westfalen. Schriftenreihe Medienforschung der LfM, Bd. 64. Berlin: Vistas.

Clausen, M. (2002). Unterrichtsqualität: Eine Frage der Perspektive? Empirische Analysen zur Übereinstimmung, Konstrukt- und Kriteriumsvalidität (Pädagogische Psychologie und Entwicklungspsychologie, Bd. 29). Zugl.: Berlin, Freie Univ., Diss., 2000. Münster: Waxmann.

Drossel, K., Eickelmann, B., \& Gerick, J. (2017). Predictors of teachers' use of ICT in school-The relevance of school characteristics, teachers' attitudes and teacher collaboration. Education and Information Technologies, 22(2), 551-573. https://doi.org/10.1007/s10639-016-9476-y.

Drossel, K., Eickelmann, B., Schaumburg, H., \& Labusch, A. (2019). Nutzung digitaler Medien und Prädiktoren aus der Perspektive der Lehrerinnen und Lehrer im internationalen Vergleich. In B. Eickelmann, W. Bos, J. Gerick, F. Goldhammer, H. Schaumburg \& M. Senkbeil, et al. (Hrsg.), ICILS 2018 \#Deutschland. Computer- und informationsbezogene Kompetenzen von Schülerinnen und Schülern im zweiten internationalen Vergleich und Kompetenzen im Bereich Computational Thinking (S. 205-240). Münster: Waxmann.

Dubberke, T., Kunter, M., McElvany, N., Brunner, M., \& Baumert, J. (2008). Lerntheoretische Überzeugungen von Mathematiklehrkräften. Zeitschrift für Pädagogische Psychologie, 22(34), 193-206. https://doi.org/10.1024/1010-0652.22.34.193.

Eccles, J.S. (2005). Subjective task value and the Eccles et al. model of achievement-related choices. In A. J. Elliot \& C.S. Dweck (Hrsg.), Handbook of competence and motivation. New York: Guilford.

Eccles, J.S., Adler, T.E., Futterman, R., Goff, S. B., Kaczala, C. M., Meece, J.L., \& Midgley, C. (1983). Expectancies, values, and academic behaviors. In J. T. Spence (Hrsg.), Achievement and achievement motives. Psychological and sociological approaches. A series of books in psychology. (S. 75-146). San Francisco: W.H. Freeman.

Eickelmann, B. (2010). Individualisieren und Fördern mit digitalen Medien im Unterricht als Beitrag zu einem förderlichen Umgang mit Heterogenität. In B. Eickelmann (Hrsg.), Bildung und Schule auf dem Weg in die Wissensgesellschaft (S. 41-56). Münster: Waxmann.

Eickelmann, B., \& Gerick, J. (2020). Lernen mit digitalen Medien. In D. Fickermann \& B. Edelstein (Hrsg.), „Langsam vermisse ich die Schule ... “ (S. 153-162). Münster: Waxmann. https://doi.org/10. 31244/9783830992318.09. 
Eickelmann, B., Lorenz, R., \& Endberg, M. (2017). Lernaktivitäten mit digitalen Medien im Fachunterricht der Sekundarstufe I im Bundesländervergleich mit besonderem Fokus auf MINT-Fächer. In R. Lorenz, W. Bos, M. Endberg, B. Eickelmann, S. Grafe \& J. Vahrenhold (Hrsg.), Schule digital der Länderindikator 2017. Schulische Medienbildung in der Sekundarstufe I mit besonderem Fokus auf MINT-Fächer im Bundesländervergleich und Trends von 2015 bis 2017 (1. Aufl. S. 231-260). Münster: Waxmann.

Eid, M., \& Schmidt, K. (2014). Testtheorie und Testkonstruktion (1. Aufl.). Psychlehrbuch plus. Göttingen: Hogrefe.

Ertmer, P.A., Ottenbreit-Leftwich, A.T., Sadik, O., Sendurur, E., \& Sendurur, P. (2012). Teacher beliefs and technology integration practices: Aa critical relationship. Computers \& Education, 59(2), 423-435. https://doi.org/10.1016/j.compedu.2012.02.001.

Faber, M. (2020). Effects of digital formative assessment tools on teaching quality and student achievement. Enschede: University of Twente. https://doi.org/10.3990/1.9789036550147.

Gerick, J., Eickelmann, B., \& Labusch, A. (2019). Schulische Prozesse als Lern- und Lehrbedingungen in den ICILS-2018-Teilnehmerländern. In B. Eickelmann, W. Bos, J. Gerick, F. Goldhammer, H. Schaumburg \& K. Schwippert, et al. (Hrsg.), ICILS 2018 \#Deutschland. Computer- und informationsbezogene Kompetenzen von Schülerinnen und Schülern im zweiten internationalen Vergleich und Kompetenzen im Bereich Computational Thinking (1. Aufl. S. 173-203). Münster: Waxmann.

Gil-Flores, J., Rodríguez-Santero, J., \& Torres-Gordillo, J.-J. (2017). Factors that explain the use of ICT in secondary-education classrooms: the role of teacher characteristics and school infrastructure. Computers in Human Behavior, 68, 441-449. https://doi.org/10.1016/j.chb.2016.11.057.

Guggemos, J., \& Seufert, S. (2021). Teaching with and teaching about technology-Evidence for professional development of in-service teachers. Computers in Human Behavior, 115, 106613. https://doi. org/10.1016/j.chb.2020.106613.

Hatlevik, O. (2017). Examining the relationship between teachers' self-efficacy, their digital competence, strategies to evaluate information, and use of ICT at school. Scandinavian Journal of Educational Research, 61(5), 555-567. https://doi.org/10.1080/00313831.2016.1172501.

Hatlevik, I., \& Hatlevik, O. (2018). Examining the relationship between teachers' ICT self-efficacy for educational purposes, collegial collaboration, lack of facilitation and the use of ICT in teaching practice. Frontiers in Psychology, 9, 935. https://doi.org/10.3389/fpsyg.2018.00935.

Heinen, R., \& Kerres, M. (2015). Individuelle Förderung mit digitalen Medien. Handlungsfelder für die systematische, lernförderliche Integration digitaler Medien in Schule und Unterricht. Gütersloh: Bertelsmann Stiftung.

Heldt, M., Lorenz, R., \& Eickelmann, B. (2020). Relevanz schulischer Medienkonzepte als Orientierung für die Schule im Zuge der fortschreitenden Digitalisierung. Unterrichtswissenschaft. https://doi.org/ 10.1007/s42010-020-00070-y.

Hew, K. F., \& Brush, T. (2007). Integrating technology into K-12 teaching and learning: current knowledge gaps and recommendations for future research. Educational Technology Research and Development, 55(3), 223-252. https://doi.org/10.1007/s11423-006-9022-5.

Hillmayr, D., Reinhold, F., Ziernwald, L., \& Reiss, K. (2017). Digitale Medien im mathematisch-naturwissenschaftlichen Unterricht der Sekundarstufe. Einsatzmöglichkeiten, Umsetzung und Wirksamkeit. Münster, New York: Waxmann.

Hu, L., \& Bentler, P. M. (1999). Cutoff criteria for fit indexes in covariance structure analysis: conventional criteria versus new alternatives. Structural Equation Modeling: A Multidisciplinary Journal, 6(1), 1-55. https://doi.org/10.1080/10705519909540118.

Inan, F. A., \& Lowther, D. L. (2010). Laptops in the K-12 classrooms: exploring factors impacting instructional use. Computers \& Education, 55(3), 937-944. https://doi.org/10.1016/j.compedu.2010.04.004.

Irion, T., \& Scheiter, K. (2018). Didaktische Potenziale digitaler Medien. Der Einsatz digitaler Technologien aus grundschul- und mediendidaktischer Sicht. Grundschule aktuell: Zeitschrift des Grundschulverbandes, 14(2), 8-11.

Kerres, M. (2018). Mediendidaktik. Konzeption und Entwicklung digitaler Lernangebote. Berlin: de Gruyter. https://doi.org/10.1515/9783110456837.

Klassen, R. M., \& Chiu, M. M. (2010). Effects on teachers' self-efficacy and job satisfaction: teacher gender, years of experience, and job stress. Journal of Educational Psychology, 102(3), 741-756. https:// doi.org/10.1037/a0019237.

Klieme, E. (2006). Empirische Unterrichtsforschung: aktuelle Entwicklungen, theoretische Grundlagen und fachspezifische Befunde. Einführung in den Thementeil. Zeitschrift für Pädagogik, 52(6), $765-773$.

Klieme, E. (2019). Unterrichtsqualität. In M. Harring, C. Rohlfs \& M. Gläser-Zikuda (Hrsg.), Handbuch Schulpädagogik. UTB, (Bd. 8698, S. 393-408). Münster: Waxmann. 
Klieme, E. (2020). Guter Unterricht - auch und besonders unter Einschränkungen der Pandemie? In D. Fickermann \& B. Edelstein (Hrsg.), „Langsam vermisse ich die Schule ... “ (S. 117-135). Münster: Waxmann. https://doi.org/10.31244/9783830992318.07.

Knezek, G., \& Christensen, R. (2016). Extending the will, skill, tool model of technology integration. Adding pedagogy as a new model construct. Journal of Computing in Higher Education, 28(3), 307-325. https://doi.org/10.1007/s12528-016-9120-2.

Kornilov, V.S., \& Khanina, I.A. (2020). Development of ICT competence in high school students when teaching physics using digital laboratories. RUDN Journal of Informatization in Education, 17(2), 146-152. https://doi.org/10.22363/2312-8631-2020-17-2-146-152.

Krumsvik, R. J. (2014). Teacher educators' digital competence. Scandinavian Journal of Educational Research, 58(3), 269-280. https://doi.org/10.1080/00313831.2012.726273.

Kunter, M., Tsai, Y.-M., Klusmann, U., Brunner, M., Krauss, S. \& Baumert, J. (2008). Students' and mathematics teachers' perceptions of teacher enthusiasm and instruction. Learning and Instruction, 18(5), 468-482. https://doi.org/10.1016/j.learninstruc.2008.06.008.

Levy, M. (2009). Technologies in use for second language learning. The Modern Language Journal, 93 , 769-782. https://doi.org/10.1111/j.1540-4781.2009.00972.x.

Li, Y., Garza, V., Keicher, A., \& Popov, V. (2019). Predicting high school teacher use of technology: pedagogical beliefs, technological beliefs and attitudes, and teacher training. Technology, Knowledge and Learning, 24(3), 501-518. https://doi.org/10.1007/s10758-018-9355-2.

Lipowsky, F. (2006). Auf den Lehrer kommt es an. Empirische Evidenzen für Zusammenhänge zwischen Lehrerkompetenzen, Lehrerhandeln und dem Lernen der Schüler. In C. Allemann-Ghionda (Hrsg.), Kompetenzen und Kompetenzentwicklung von Lehrerinnen und Lehrern. Ausbildung und Beruf. Zeitschrift für Pädagogik. Beiheft, (Bd. 51, S. 47-70). Weinheim: Beltz.

Lipowsky, F. (2015). Unterricht. In E. Wild \& J. Möller (Hrsg.), Pädagogische Psychologie (S. 69-105). Berlin, Heidelberg: Springer.

Lucas, M., Bem-Haja, P., Siddiq, F., Moreira, A., \& Redecker, C. (2021). The relation between in-service teachers' digital competence and personal and contextual factors: what matters most? Computers \& Education, 160, 104052. https://doi.org/10.1016/j.compedu.2020.104052.

López-Vargas, O., Duarte-Suárez, L., \& Ibáñez-Ibáñez, J. (2017). Teacher's computer self-efficacy and its relationship with cognitive style and TPACK. Improving Schools, 20(3), 264-277. https://doi.org/10. $1177 / 1365480217704263$.

Mishra, P., \& Koehler, M. J. (2006). Technological pedagogical content knowledge. A framework for teacher knowledge. Teachers College Record, 108(6), 1017-1054. https://doi.org/10.1111/j.1467-9620. 2006.00684.x.

Muthén, L. K. \& Muthén, B. O. (1998-2016). Mplus 8.1 [Computer software]. Los Angeles, CA: Muthén \& Muthén.

Peterson, R. A. \& Brown, S. P. (2005). On the Use of Beta Coefficients in Meta-Analysis. Journal of Applied Psychology, 90(1), 175-181.

Petko, D. (2012). Hemmende und förderliche Faktoren des Einsatzes digitaler Medien im Unterricht: Empirische Befunde und forschungsmethodische Probleme. In R. Schulz-Zander, B. Eickelmann, H. Moser, H. Niesyto \& P. Grell (Hrsg.), Jahrbuch Medienpädagogik 9. Jahrbuch Medienpädagogik, (Bd. 9.2012, S. 29-50). Wiesbaden: Springer VS.

Praetorius, A.-K., Klieme, E., Herbert, B., \& Pinger, P. (2018). Generic dimensions of teaching quality: the German framework of three basic dimensions. ZDM, 50(3), 407-426. https://doi.org/10.1007/ s11858-018-0918-4.

Praetorius, A.-K., Vieluf, S., Saß, S., Bernholt, A., \& Klieme, E. (2016). The same in German as in English? Investigating the subject-specificity of teaching quality. Zeitschrift für Erziehungswissenschaft, 19(1), 191-209. https://doi.org/10.1007/s11618-015-0660-4.

Rahimi, M., \& Yadollahi, S. (2011). Computer anxiety and ICT integration in English classes among Iranian EFL teachers. Procedia Computer Science, 3, 203-209. https://doi.org/10.1016/j.procs.2010. 12.034.

Rakoczy, K. \& Klieme, E. (2005). Befragungsinstrumente (Materialien zur Bildungsforschung, Bd. 13). Frankfurt am Main: GFPF. Verfügbar unter: http://nbn-resolving.de/urn:nbn:de:0111-opus-31060

Rohatgi, A., Scherer, R., \& Hatlevik, O. (2016). The role of ICT self-efficacy for students' ICT use and their achievement in a computer and information literacy test. Computers \& Education, 102, 103-116. https://doi.org/10.1016/j.compedu.2016.08.001.

Rubach, C., \& Lazarides, R. (2019). Eine Skala zur Selbsteinschätzung digitaler Kompetenzen bei Lehramtsstudierenden. Entwicklung eines Instrumentes und die Validierung durch Konstrukte zur Mediennutzung und Werteüberzeugungen zur Nutzung digitaler Medien im Unterricht. Zeitschrift für Bildungsforschung, 2(78), 4. https://doi.org/10.1007/s35834-019-00248-0. 
Rubach, C., \& Lazarides, R. (2021a). Addressing 21st-century digital skills in schools-Development and validation of an instrument to measure teachers' basic ICT competence beliefs. Computers in Human Behavior, 115, 106636. https://doi.org/10.1016/j.chb.2020.106636.

Rubach, C., \& Lazarides, R. (2021b). Bedingungen für die Umsetzung motivationsförderlicher Unterrichtsstrategien durch digitale Medien. In R. Lazarides \& D. Raufelder (Hrsg.), Motivation in unterrichtlichen Lehr-Lernkontexten. Perspektiven aus Pädagogik, Psychologie und Fachdidaktiken. Edition ZfE, (Bd. 10, S. 435-461). Wiesbaden: Springer VS.

Rubach, C., Lazarides, R., Brendel, N., \& Krauskopf, K. (2019). Learning by doing. Eine explorative Erhebung zur Förderung digitaler Kompetenzeinschätzungen durch die Verwendung digitaler Medien in der Hochschuldidaktik. In T. Ehmke, P. Kuhl \& M. Pietsch (Hrsg.), Lehrer. Bildung. Gestalten. Beiträge zur empirischen Forschung in der Lehrerbildung (S. 164-175). Weinheim: Juventa.

Sabanci, A., Ozyildirim, G., \& Imsir, R. (2014). The effect of ICT usage on the classroom management: a case study in language teaching. International Review of Social Sciences and Humanities, 7(1), 232-245.

Sailer, M., Stadler, M., Schultz-Pernice, F., Franke, U., Schöffmann, C., Paniotova, V., et al. (2021). Technology-related teaching skills and attitudes: validation of a scenario-based self-assessment instrument for teachers. Computers in Human Behavior, 115, 106625. https://doi.org/10.1016/j.chb.2020. 106625.

Schaumburg, H. (2017). Chancen und Risiken digitaler Medien in der Schule. Medienpädagogische und -didaktische Perspektiven. In Bertelsmann Stiftung (Hrsg.), Individuell fördern mit digitalen Medien. Chancen, Risiken, Erfolgsfaktoren (2. Aufl. S. 20-94). Gütersloh: Bertelsmann.

Schaumburg, H., Gerick, J., Eickelmann, B., \& Labusch, A. (2019). Nutzung digitaler Medien aus der Perspektive der Schülerinnen und Schüler im internationalen Vergleich. In B. Eickelmann, W. Bos, J. Gerick, F. Goldhammer, H. Schaumburg \& M. Senkbeil, et al. (Hrsg.), ICILS 2018 \#Deutschland. Computer- und informationsbezogene Kompetenzen von Schülerinnen und Schülern im zweiten internationalen Vergleich und Kompetenzen im Bereich Computational Thinking. Münster: Waxmann.

Scherer, R., Tondeur, J., \& Siddiq, F. (2017). On the quest for validity: testing the factor structure and measurement invariance of the technology-dimensions in the Technological, Pedagogical, and Content Knowledge (TPACK) model. Computers \& Education, 112, 1-17. https://doi.org/10.1016/j.compedu. 2017.04.012.

Schmid, U., Goertz, L., \& Behrens, J. (2017). Monitor Digitale Bildung. Die Schulen im digitalen Zeitalter. Gütersloh: Bertelsmann.

Siddiq, F., Hatlevik, O., Olsen, R. V., Throndsen, I., \& Scherer, R. (2016). Taking a future perspective by learning from the past-A systematic review of assessment instruments that aim to measure primary and secondary school students' ICT literacy. Educational Research Review, 19, 58-84. https://doi. org/10.1016/j.edurev.2016.05.002.

Siemsen, E., Roth, A., \& Oliveira, P. (2010). Common method bias in regression models with linear, quadratic, and interaction effects. Organizational Research Methods, 13(3), 456-476. https://doi.org/ $10.1177 / 1094428109351241$.

Sieve, B.F. (2015). Interaktive Tafeln im naturwissenschaftlichen Unterricht. Entwicklung und Evaluation einer Fortbildungsmaßnahme für Chemielehrkräfte. Wiesbaden: Springer Spektrum. https://doi.org/ 10.1007/978-3-658-09946-6.

Sipilä, K. (2011). No pain, no gain? Teachers implementing ICT in instruction. Interactive Technology and Smart Education, 8(1), 39-51. https://doi.org/10.1108/17415651111125504.

Sundqvist, K., Korhonen, J., \& Eklund, G. (2021). Predicting Finnish subject-teachers' ICT use in home economics based on teacher- and school-level factors. Education Inquiry, 12(1), 73-93. https://doi. org/10.1080/20004508.2020.1778609.

Tezci, E. (2010). Attitudes and knowledge level of teachers in ICT use: the case of Turkish teachers. Journal of Human Sciences, 7(2), 19-44.

Voss, T., \& Wittwer, J. (2020). Unterricht in Zeiten von Corona: Ein Blick auf die Herausforderungen aus der Sicht von Unterrichts- und Instruktionsforschung. Unterrichtswissenschaft, 48(4), 601-627. https://doi.org/10.1007/s42010-020-00088-2.

Ware, P., Liaw, M.-L., \& Warschauer, M. (2012). The use of digital media in teaching English as an international language. In L. Alsagoff, S. Lee McKay, G. Hu \& W. A. Renandya (Hrsg.), Principles and practices for teaching English as an international language (S. 67-84). New York: Routledge.

Wetterich, F., Burghart, M. \& Rave, N. (2014). Medienbildung an deutschen Schulen Handlungsempfehlungen für die digitale Gesellschaft. Berlin: atene $\mathrm{KOM} \mathrm{GmbH}$.

Xu, Y., Wang, D., Collins, P., Lee, H., \& Warschauer, M. (2021). Same benefits, different communication patterns: comparing children's reading with a conversational agent vs. a human partner. Computers \& Education, 161, 104059. https://doi.org/10.1016/j.compedu.2020.104059. 